\title{
Color embeddings, charge assignments, and proton stability in unified gauge theories*
}

\author{
M. Gell-Mann, ${ }^{*}$ P. Ramond, ${ }^{* *}$ and R. Slansky \\ Theoretical Division, University of California, Los Alamos Scientific Laboratory, Los Alamos, New \\ Mexico 87545
}

Three problems in hypothetical unified theories of electromagnetic, weak, and strong interactions are discussed here. First, the problem of embedding color in any simple gauge group is solved, and a complete classification of theories where the fermion color is restricted to $\mathbf{1}^{c}, \mathbf{3}^{c}$, and $\overline{3}^{c}$ of $\mathrm{SU}_{3}^{c}$ is given. Generalizations are also indicated. Second, an unbroken $U_{1}$ generated by electric charge is embedded into each of the above theories and the charge assignments analyzed. Third, the general problem of stabilizing the proton by a suitable atomic mass number $A$ is studied for the same set of theories. It is always possible to define $A$ if the gauge group is not too small. In many of these theories there must be fermions with weird values of $A$ : leptons with $A \neq 0$ and quarks with $A \neq 1 / 3$. Examples are discussed. Some future directions of research are indicated.

\section{CONTENTS}

I. Introduction

II. Embedding $\mathrm{SU}_{3}^{c}$ in Simple Lie Groups

A. Unitary groups

B. Orthogonal groups

C. Symplectic groups

D. Exceptional groups

III. The Electric Charge Operator

IV. Baryon Number Conservation

A. Double f, Majorana mass breaking

B. Single $f$, doubling and breaking elsewhere

Acknowledgments

Appendix

References

721

732

733

734

735

736

736

739

740

741

742

742

743

\section{INTRODUCTION}

Quantum field theory with local gauge invariance appears to provide the appropriate framework for a dynamical theory of all elementary particle interactions. It is well known that electromagnetic interactions were the first to be described this way: the local phase invariance of the Lagrangian allows the construction of a renormalizable quantum field theory that agrees impressively well with experiment. Local phase invariance was generalized to non-Abelian gauge groups by Yang and Mills (1954) and Shaw (1955). [Also see Utiyama (1956), GellMann and Glashow (1961), and Kibble (1961).] Although Yang-Mills theories were immediately seen to be mathematically beautiful and physically suggestive, it was not clear how to construct a sensible model in which the vector bosons (other than the photon) acquire masses without destroying its (then hoped for) renormalizability. Then it was discovered by Higgs $(1964 \mathrm{a}, \mathrm{b})$ and others that the spontaneous breaking of a local symmetry does not imply a zero-mass Goldstone boson as it does in

*Work supported by the U. S. Energy. Research and Development Administration under contract No. W-7405-ENG-36 and contract No. EY76-C-03-0068, and by the Alfred P. Sloan Foundation.

$\dagger$ Permánent address: Lauritsen Laboratory, California Institute of Technology, Pasadena, California 91125.

\$Robert Andrews Millikan Senior Research Fellow.

${ }^{1}$ The Goldstone theorem is discussed in Goldstone (1961); Nambu and Jona-Lasinio (1961); and Goldstone, Salam, and Weinberg (1962). For the Higgs mechanism, see Higgs (1964a, b); Englert and Brout (1964); and Guralnik, Hagen, and Kibble (1964). conventional theories. ${ }^{1}$ When the quadratic part of the Lagrangian is rediagonalized by a gauge transformation, the degree of freedom that was expected to be the Goldstone boson becomes the longitudinal-spin degree of freedom of a massive vector boson. Later t'Hooft (1971) provided the crucial proof that spontaneously broken Yang-Mills theories are indeed renormalizable.

By extending the local gauge invariance beyond the $U_{1}$ of electrodynamics, it became possible to construct sensible models of weak and electromagnetic interactions in which the weak bosons acquire large masses (of order $100 \mathrm{GeV}$ ) through the Higgs mechanism (Weinberg, 1967; Salam, 1968). The experimental existence of the weak charged and neutral currents along with the electromagnetic current implies a local symmetry group at least as large as $\mathrm{SU}_{2} \times \mathrm{U}_{1}$ (Glashow, 1959). The basic strategy of using Yang-Mills Lagrangians to unify electromagnetic and weak interactions is very attractive. However, the $\mathrm{SU}_{2} \times \mathrm{U}_{1}$ theory is somewhat awkward. Besides the two gauge couplings of the two simple factors of the gauge group, there are choices of particle fields, their representations, and other arbitrary parameters in the $\mathrm{SU}_{2} \times \mathrm{U}_{1}$ invariant Lagrangian. It also ignores the strong interactions. Nevertheless, this model has provided a framework for organizing huge quantities of experimental data.

The formulation of a Yang-Mills theory of the strong interactions is greatly simplified once the fundamental role of quarks and gluons is recognized. The proposal subscribed to in this paper is that of quantum chromodynamics $(\mathrm{QCD})^{2}$; the strong-interaction gauge group is called the color group. The gauged color symmetry must be at least as large as $\mathrm{SU}_{3}$. (The choice of $\mathrm{SU}_{3}$

${ }^{2} \mathrm{QCD}$ was originally introduced by Nambu (1966) in a version based on the unconfined, integrally charged quarks of Han and Nambu (1965). Fractionally charged quarks were usually assigned parastatistics, as described by Greenberg (1964). Later it was shown by Fritzsch and Gell-Mann (1971) and Bardeen, Fritzsch, and Gell-Mann (1972) that the concept of color, with isolated particles assumed to be restricted to color singlets, had the same effect as parastatistics, with isolated particles assumed to be restricted to bosons and fermions. For early discussions of QCD with confined color see Fritzsch and GellMann (1972); Fritzsch, Gell-Mann, and Leutwyler (1973); and Weinberg $(1973 a, b)$. Meanwhile, the asymptotic freedom of QCD was being pointed out by Politzer (1973) and by Gross and Wilczek (1973). 
solves the statistics problem of the baryonic ground state, gives the correct $\pi^{0}$ decay rate with fractionally charged quarks, and yields rough agreement with the hadron-muon ratio $R$ in $e^{+} e^{-}$annihilation.) In QCD the $\mathrm{SU}_{3}^{c}$ is unbroken, the theory is asymptotically free, and color is conjectured to be confined. (Color confinement may in fact be only approximate, but it is conceptually simplest for us to assume here that only color singlet states are observed in Nature.)

We neglect the effects of gravity in this paper, although there are some brief comments on supersymmetry and supergravity near the end of this section.

Any hypothetical unified Yang-Mills theory of electromagnetic, weak, and strong interactions must have a local symmetry group $G$ at least as large as $G^{w} \times \mathrm{SU}_{3}^{c}$ :

$$
G \supseteq G^{w} \times \mathrm{SU}_{3}^{c},
$$

where $G^{w} \supseteq \mathrm{SU}_{2} \times \mathrm{U}_{1}$ includes the low-mass bosons of the weak interactions and the photon. We assume that the photon couples to one of the $G^{w}$ currents and, of course, that the corresponding $U_{1}$ is unbroken. In the minimal scheme $\mathrm{SU}_{2} \times \mathrm{U}_{1} \times \mathrm{SU}_{3}$, there are three independent coupling constants and much arbitrariness in assigning fermions and other particles to representations of $G$. From a theoretical viewpoint, this looseness in the formulation of the theory would seem to be an unacceptable price to pay, for example, for economizing on the number of vector bosons. The simplest proposal for improving on this situation is to enlarge $G$ so that there is only one gauge coupling constant (Georgi and Glashow, 1974; Fritzsch and Minkowski, 1975) and so that all the elementary fermions (quarks and leptons) are contained in a few ir reducible representations of $G$. In the last of these references, the authors examined many of the cases treated here, but our discussion is somewhat more systematic.

It is assumed in this paper that $G$ is a Lie group (and not, for example, a graded Lie group), that $G$ contains $\mathrm{SU}_{2} \times \mathrm{U}_{1} \times \mathrm{SU}_{3}^{c}$, and that there is only one independent gauge coupling. Thus $G$ must be simple, or semisimple in the form $G \times G$ ( $G$ simple) where some global reflection symmetry constrains the (unrenormalized) gauge coupling constants to the two simple factors to be equal. The requirement that $G$ be simple fixes many parameters and relationships that need to be determined experimentally in the $\mathrm{SU}_{2} \times \mathrm{U}_{1} \times \mathrm{SU}_{3}^{c}$ model, but it also implies new interactions of Nature that have not been observed.

For the moment we assume the full gauge group $G$ is simple, and leave until later the generalization to $G \times G$ theories. The flavor group $G^{\mathrm{f1}}$, which is generated by the currents that couple to the color singlet bosons, must include $G^{w}: G^{\mathrm{f} 1} \supseteq G^{w}$. Thus $G$ has a maximal subgroup decomposition of the form,

$$
G \supset G^{\mathrm{f} 1} \times \mathrm{SU}_{3}^{c} \text {. }
$$

One purpose of this paper is to list the embeddings of $\mathrm{SU}_{3}^{c}$ in $G$ and to classify the structure of $G^{\mathrm{f1}}$. We note here that if $G^{\mathrm{f1}}$ is larger than $\mathrm{SU}_{2} \times \mathrm{U}_{1}$, then the new generators are coupled to bosons that mediate interactions not yet observed. The supplementary flavor bosons are either of high mass or else not coupled significantly to transitions between light, familiar parti- cles. Other new bosons implied by this kind of unification can include diotons, which are color octets that also carry flavor; leptoquarks, which change quarks into leptons; and diquarks, which change quarks into antiquarks. Clearly an unstable proton is often predicted in this kind of unified theory. Much of our paper is devoted to that problem.

The fields appearing in the Lagrangian are assigned to representations of $G$. The spin $1 / 2$ fermion representation $f$ must include leptons $\left(1^{c}\right)$, quarks $\left(3^{c}\right)$, and antiquarks $\left(\overline{3}^{c}\right)$ : at present experiment does not suggest the existence of new fermions transforming as higher $\mathrm{SU}_{3}^{c}$ representations. Theoretically there is no objection to having fermions in more complicated color representations; indeed such fermions are commonplace in supersymmetric theories. Nevertheless, it is usually the case that some set of fermions (which might have spin $3 / 2$, for example) belong to a representation containing only $1^{c}, 3^{c}$ and $\overline{3}^{c}$. In this article, we usually make the more limiting assumption that the spin $1 / 2$ fermion colors are actually restricted to $1^{c}, 3^{c}$ and $\overline{3}^{c}$. The problem of finding all embeddings of $\mathrm{SU}_{3}^{c}$ in any simple $G$ larger than $\mathrm{SU}_{2} \times U_{1} \times \mathrm{SU}_{3}^{c}$, with the proviso that there exists at least one nontrivial representation containing at most $1^{c}, 3^{c}$ and $\overline{3}^{c}$, is solved in Sec. II. We also discuss the structure of $G^{\mathrm{f} 1}$ and find all other representations of $G$ satisfying the same color restriction. These results will now be reviewed in detail; Sec. II may be regarded as a mathematical appendix.

The embedding procedure is greatly simplified by a theorem that is proved in the Appendix: if for a given embedding of $\mathrm{SU}_{3}^{c}$ in $G$, the color content of any nontrivial representation $\mathrm{f}$ is restricted to $1^{c}, 3^{c}$, and $\overline{3}^{c}$, then the "fundamental" representation of $G$ must also contain at most, $1^{c}, 3^{c}$, and $\overline{3}^{c}$. By the fundamental representation we mean the defining representations of the classical groups and the lowest-dimensional nontrivial representations of the exceptional groups. Of course, the color restriction on $f$ and the fundamental representation can be loosened to include $6^{c}, \overline{6}^{c}, 8^{c}$, etc., if desired, but this is not done here. The embedding is then identified by constructing the adjoint representation, which is always easily obtained from the fundamental one. Since the embedding is of the form $G \supset G^{\mathrm{f1}} \times \mathrm{SU}_{3}^{c}$, the color singlet part of the adjoint representation then identifies the flavor group, and the remaining bosons are also listed. We construct all other irreducible representations of $G$ with the color restriction and put the fermions into these representations or direct sums of them.

The structure of $G^{\mathrm{fl}}$ falls into one of four categories. We summarize the results of Sec. II in terms of those categories.

Class $I: \quad G^{\mathrm{f} 1}=G_{l} \times G_{q} \times \mathrm{U}_{1}$, where $G_{l}$ is a nontrivial simple factor that transforms only the color singlets, and $G_{q}$ is another nontrivial simple factor that transforms only the color triplets (and antitriplets) of the fundamental representation. ${ }^{3}$ The $U_{1}$ distinguishes $1^{c}$ from $3^{c}$ and (or) $\overline{3}^{c}$. This embedding occurs only if $G$ is a classical group, i.e., if $G$ is $\mathrm{SU}_{n}$ (unitary), $\mathrm{SO}_{n}$

\footnotetext{
${ }^{3} \mathrm{We}$ often discuss $\mathrm{SO}_{4} \approx \mathrm{SU}_{2} \times \mathrm{SU}_{2}$ as if it were a simple (sub) group.
} 
(orthogonal), or $S p_{2 n}$ (symplectic). Only the fundamental representation of $G$ satisfies the color restriction: $\mathbf{n}$ of $\mathrm{SU}_{n} ; \mathbf{n}$ of $\mathrm{SO}_{n}$ (also called the vector representation $\mathbf{v}$ ); or the $2 \mathrm{n}$ of $S p_{2 n}$. Thus the color singlets of the fundamental representation may be identified with the leptons, and the color triplets with quarks. (See Cases 1, 4, and 6 in Sec. II.) Since the quarks and leptons have commuting flavor groups, the observed universality of leptonic and quark electromagnetic and weak charges must come from the symmetry-breaking mechanism. The $\mathrm{n}$ of $\mathrm{SO}_{n}$ and the $2 \mathrm{n}$ of $S p_{2 n}$ are self-conjugate representations, and the refore contain equal numbers of $3^{c}$ and $\overline{3}^{c}$. In this embedding the $\mathrm{n}$ of $\mathrm{SU}_{n}$, which is complex, contains $1^{c}$ and $3^{c}$ only.

Class II: $G^{\mathrm{f} 1}=G_{l} \times G_{q} \times G_{\bar{\gamma}} \times \mathrm{U}_{1} \times \mathrm{U}_{1}$. This is possible only for $G=\mathrm{SU}_{n}$, with fermions assigned to the $\mathrm{n}$, where n contains $3^{c} q$-type quarks, and $\overline{3}^{c} \bar{r}$-type antiquarks. The two $\mathrm{U}_{1}$ 's distinguish among $1^{c}, 3^{c}$, and $\overline{3}^{c}$. This embedding is quite similar to Class I, but it contains some additional interest because there is a temptation to enlarge the color group to $\mathrm{SU}_{3} \times \mathrm{SU}_{3}$. (See Sec. II, Case 3.)

Class III: $G^{\mathrm{f1}}=G_{\alpha+l} \times \mathrm{U}_{1}$, where $G_{\alpha+l}$ transforms the color singlet piece of the fundamental representation, but the fermions are in a different representation such that the same simple ${ }^{3}$ factor $G_{q+l}$ transforms both quarks and leptons. There are two cases:

If $G=\mathrm{SU}_{n}$, then the fermions may be assigned to the irreducible representations constructed from $(\mathbf{n} \times \mathbf{n}$ $\times \ldots \times \mathbf{n})_{A}$, where ()$_{A}$ means to antisymmetrize the Kronecker product. Except for $\left(\mathrm{n}^{n / 2}\right)_{A}, n$ even, these representations are all complex. (See Case 2 in Sec. II.)

If $G=\mathrm{SO}_{m}$, f may be a spinor representation. There is one self-conjugate spinor for $\mathrm{SO}_{2 n+1}$ of dimension $2^{n}$. $\mathrm{SO}_{4 n}$ has two inequivalent self-conjugate spinors, each of dimension $2^{2 n-1}$, and the two spinors of $\mathrm{SO}_{4 n+2}$ are complex and conjugate to one another. Each has dimension $2^{2 n}$. (See Case 5.)

In this class the universality of the weak charges is natural since there is only one non-Abelian factor in $G^{\mathrm{f} 1}$. However, the relation between quark and lepton electric charges has to come from the symmetry breaking.

Class $I V: G^{\mathrm{f} 1}=G_{q+\imath}$. This embedding, which contains no $U_{1}$ factor that distinguishes $1^{c}$ from $3^{c}$, is possible only for the exceptional groups. Three of the five exceptional groups satisfy our assumptions, and in each case, only the fundamental representation satisfies the color restriction. We enumerate the results. (See Cases 7, 8, and 9.) For $F_{4}$, where $G^{\mathrm{f1}}=\mathrm{SU}_{3}$, the fundamental representation is the 26, which is self-conjugate. The flavor group for $E_{6}$ is $\mathrm{SU}_{3} \times \mathrm{SU}_{3}$ and the fermions are assigned to the 27, which is complex. For $E_{7}, G^{\mathrm{f1}}=\mathrm{SU}_{6}$ and the fermions are assigned to the 56 , which is self-conjugate.

The restrictiveness of the exceptional groups, both in number and in internal structure, makes them quite attractive for model building. The universality of the quark and leptonic weak and electromagnetic charges is a consequence of the group structure, as is the $1 / 3$ integral charge structure of the quarks if the leptons have charges \pm 1 and 0 only. The $\mathrm{SU}_{3}^{c}$ is naturally embedded in these groups (Günaydin and Gürsey, 1973; Gürsey, 1976).

The results of our classification are summarized in Table I. There are no other embeddings of $\mathrm{SU}_{3}^{c}$ in any simple $G$ for which there is at least one representation satisfying the $1^{c}, 3^{c}, \overline{3}^{c}$ color restriction.

The classification of the fermion representations is not complete until we analyze their helicity structure. Fermions of a given chirality are transformed among themselves under $G$, which we continue to assume to be simple.

We first study the case where a scalar fermion number is defined, so that fermions and antifermions are initially distinguished. Suppose the left-handed fermions are assigned to $f_{L}$ of $G$ and the right-handed fermions to $\mathbf{f}_{R}$ of $G$. Then all left-handed states are in $\mathbf{f}_{L}+\overline{\mathbf{f}}_{R}$, and all right-handed states are in $\mathbf{f}_{R}+\bar{f}_{L}$. If the quark-gluon couplings are to conserve parity, there must be a discrete symmetry that relates the quarks in $f_{L}$ to those in $\mathbf{f}_{R}$, and also relates any antiquarks that may be in $\mathbf{f}_{L}$ to those in $\mathrm{f}_{R}$. This same discrete symmetry will relate the leptons in $\mathbf{f}_{L}$ and $\mathbf{f}_{R}$, if we ignore the possibility of adding $G$ singlets to either $\mathbf{f}_{L}$ or $\mathbf{f}_{R}$. Consequently, $\mathbf{f}_{L}$ and $\mathbf{f}_{R}$ are either equivalent or else related by group conjugation. Theories in which $f_{R}$ is equivalent to $f_{L}$ are called vectorlike (Georgi and Glashow, 1973). If $f_{R}$ is

TABLE I. Embedding of $\mathrm{SU}_{3}^{c}$ in $G$, representations with $1^{c}, 3^{c}, \overline{3}^{c}$ only of color. The bracket in case 5 means integer part.

\begin{tabular}{cllll}
\hline \hline Case & $G$ & \multicolumn{1}{c}{$G^{\mathrm{f1}}$} & $\mathrm{f}$ & Dimensionality \\
\hline 1. & $\mathrm{SU}_{n}$ & $\mathrm{SU}_{n_{1}} \times \mathrm{SU}_{n_{3}} \times \mathrm{U}_{1}$ & $\mathrm{n}$ & $n=n_{1}+3 n_{3}$ \\
2. & $\mathrm{SU}_{n}$ & $\mathrm{SU}_{n-3} \times \mathrm{U}_{1}$ & $\left(\mathrm{n}^{k}\right)_{A}$ & $\left(\begin{array}{l}n \\
k\end{array}\right)$ \\
3. & $\mathrm{SU}_{n}$ & $\mathrm{SU}_{n_{1}} \times \mathrm{SU}_{n_{3}} \times \mathrm{SU}_{n_{\overline{3}}} \times \mathrm{U}_{1} \times \mathrm{U}_{1}$ & $\mathrm{n}$ & $n=n_{1}+3 n_{3}+3 n_{\overline{3}}$ \\
4. & $\mathrm{SO}_{n}$ & $\mathrm{SO}_{n_{1}} \times \mathrm{SU}_{n_{3}} \times \mathrm{U}_{1}$ & $\mathrm{n}$ & $n=n_{1}+6 n_{3}$ \\
5. & $\mathrm{SO}_{n}$ & $\mathrm{SO}_{n-6} \times \mathrm{U}_{1}$ & $\sigma, \sigma^{\prime}$ & ${ }_{2}^{\left[\frac{n-1}{2}\right]}$ \\
6. & $\mathrm{Sp}_{2 n}$ & $\mathrm{Sp}_{2 n_{1}} \times \mathrm{SU}_{n_{3}} \times \mathrm{U}_{1}$ & $2 \mathrm{n}$ & $n=n_{1}+3 n_{3}$ \\
7. & $F_{4}$ & $\mathrm{SU}_{3}$ & 26 & 26 \\
8. & $E_{6}$ & $\mathrm{SU}_{3} \times \mathrm{SU}_{3}$ & 27 & 27 \\
9. & $E_{7}$ & $\mathrm{SU}_{6}$ & 56 & 56 \\
\hline \hline
\end{tabular}


equivalent to $\overline{\mathrm{f}}_{E}$, we call the theory flavor chiral. ${ }^{4}$

We now examine the case where a scalar fermion number cannot be defined; $f_{L}$ contains all the left-handed fermions and antifermions of the theory, and $f_{R}$ contains all the right-handed ones. Parity conservation in the quark-gluon couplings then implies that the quarks in $f_{L}$ and $\mathbf{f}_{R}$ are related by a discrete symmetry. As before the theory is either vectorlike or flavor chiral. In the latter case $\left(f_{R}\right.$ equivalent to $\left.\bar{f}_{L}\right)$, there exists a pseudoscalar quantum number that initially distinguishes $f_{L}$ and $\mathrm{f}_{R}$.

Further limitations on $\mathbf{f}_{R}$ given $\mathbf{f}_{\boldsymbol{L}}$ follow from the renormalizability of the theory. The theory must not have divergences due to Adler (1969), Bell and Jackiw (1969) triangle anomalies. The fermion representation falls into one of three categories (Georgi and Glashow, 1973).

(1) If $f_{L}$ is a self-conjugate representation, there will never be any problem with triangle anomalies. Such theories are always vectorlike.

(2) If $f_{L}$ is a complex representation but $G$ is not a unitary group, there is again no problem with triangle anomalies. These theories are based on $G=E_{6}(\mathrm{f}=27)$ or $\mathrm{SO}_{4 n+2}(\mathbf{f}=$ spinor $),{ }^{5}$ and may be vectorlike or flavor chiral.

(3) The complex representations of $\mathrm{SU}_{n}(n \geqslant 3)$ are unsafe, but may be used in a vectorlike theory, or in a nonvectorlike theory if there is an accidental cancellation of right- and left-handed anomalies separately. (In the latter case $f_{R}$ is equivalent to $\bar{f}_{L}$, where $f_{R}$ is reducible.) When the cancellation does take place, $f_{L}$ often appears as the branching of a safe representation of a larger group. For example, the anomalies from the $\overline{5}$ and $\mathbf{1 0}$ of $\mathrm{SU}_{5}$ cancel, where the decomposition of the spinor of $\mathrm{SO}_{10}$ into $\mathrm{SU}_{5}$ representations is given as $1+\overline{5}+10$ (Georgi, 1975). (The singlet does not contribute to the anomaly.) Such accidental cancellation is somewhat artificial and we do not consider examples of it.

The results of this classification of the chiral structure of the fermion representations are summarized in Table $\Pi$. Except for gauge groups permitting a flavor chiral theory, it is most natural to assume a vectorlike theory.

In Sec. III, which we also treat as a mathematical appendix to this introduction, we have studied the possible embeddings of the $U_{1}$ generated by the electric charge operator in $G^{\mathrm{fl}}$. Quarks are assumed to have charges in the sequence $(. .5 / 3,2 / 3,-1 / 3,-4 / 3, \ldots)$, and leptons to have integral charges. (This is a fairly weak assumption for Class IV embeddings.) The results are organized around the above classification of the structure of $G^{\mathrm{f1}}$.

In the Class I embeddings $\left(G^{\mathrm{f1}}=G_{l} \times G_{q} \times \mathrm{U}_{1}\right)$, there is

\footnotetext{
"The name "flavor chiral" is appropriate for $\mathrm{E}_{6}$ and $\mathrm{SO}_{10}$, where the flavor groups are $\mathrm{SU}_{3} \times \mathrm{SU}_{3}$ and $\mathrm{SU}_{2} \times \mathrm{SU}_{2}$, respective$1 y$, and for the simplest flavor chiral assignment the factors act chirally on the quarks. For $\mathrm{SO}_{14}, \mathrm{SO}_{18}, \ldots$, this name is less appropriate. Of course, when a scalar fermion number cannot be defined, the word vectorlike is not really appropriate either, since even in the absence of fermion masses such theories cannot be described using Dirac spinors with ordinary vector coupling.

${ }^{5}$ The proof that $E_{6}$ is safe is carried out in Gürsey, Ramond, and Sikivie (1975).
}

TABLE II. Classification of chiral fermion representations.

\begin{tabular}{lll}
\hline Type of representation & $\begin{array}{c}\mathbf{f}_{R} \sim \mathbf{f}_{L} \\
\text { (vectorlike) }\end{array}$ & \multicolumn{1}{c}{$\begin{array}{c}\mathbf{f}_{R} \sim \overline{\mathbf{f}}_{L} \\
\text { (flavor chiral) }\end{array}$} \\
\hline Real & Possible & $\begin{array}{l}\text { Identical to vectorlike } \\
\text { Complex safe }\end{array}$ \\
Complex unsafe & Possible & Possible \\
Psually not possible
\end{tabular}

${ }^{a}$ Safe and unsafe from anomalies.

great freedom in defining the electric charge operator $Q$. The only constraint arises from the tracelessness of all $G^{\mathrm{f} 1}$ generators: the sum of all fermion charges must be zero. There is no restriction on $Q$ when the fermion representation is self-conjugate under antiparticle conjugation. Results for Class II embeddings are similar.

In Class III embeddings $\left(G^{\mathrm{f} 1}=G_{q+l} \times \mathrm{U}_{1}\right)$ the quarks and leptons transform under the same simple subgroup ${ }^{3}$ of $G^{\mathrm{f} 1}$, but the extra $\mathrm{U}_{1}$ distinguishes $1^{c}$ from $3^{c}$. Nevertheless, the quark charges, assumed to be in the $-1 / 3+$ integer sequence, determine the lepton charges. Usually the sum of the quark charges does not vanish. A general parametrization of the charge operator is given in Sec. III.

In Class IV embeddings $\left(G^{\mathrm{f} 1}=G_{\alpha+l}\right)$, the sum of the quark charges is zero and the quark charges determine the lepton charges. Possible charge assignments are easily listed.

We turn now to the question of proton decay, which can often occur in unified theories since quarks and leptons both appear in the same irreducible representations of $G$, and there must be bosons and possibly other fields that mediate quark-lepton transitions. In some models the leptoquarks also couple quarks to antiquarks that are assigned to the fermion multiplet, permitting the proton to decay to meson plus lepton in second order even in the absence of symmetry violation. After the local symmetry $G$ is broken to $\mathrm{U}_{1} \times \mathrm{SU}_{3}^{c}$, the proton will certainly decay unless there remains a conservation law that prohibits it. Thus, if the proton is stable, there must be a conserved quantum number $A$ ( $A$ is a generalized atomic mass number) that agrees with the usual definition for ordinary matter, and such that the lowest-mass state with $A=1$ is the proton. The problem of defining $A$ in a Yang-Mills theory of quarks and leptons based on a simple $G$ is detailed in Sec. IV. The notation used there is that of a vectorlike theory, but we show in the examples at the end of this section that the extension to flavor chiral theories is trivial. Again we provide a full discussion here, and treat Sec. IV as a mathematical appendix.

The experimental bound on the decay rate of the proton into a muon is $\tau^{-1}<10^{-30} /$ year (Reines and Crouch, 1974), and the half-life of the proton is at least as long as $2 \times 10^{28}$ years (Gurr et al., 1967). Many theorists have simply accepted the instability of the proton in lowest order and supposed that the responsible bosons have such high effective masses (order of the Planck mass, $1.22 \times 10^{19} \mathrm{GeV}$ ) that the amplitude is reduced to an experimentally acceptable value. ${ }^{6}$ This may not be a

${ }^{6} \mathrm{~S}$. Meshkov has nicknamed these bosons "Intermediate Vector Baseballs." 
problem in principle, since the mass scale associated with a universal strength for the weak and strong interactions may be on the order of $10^{15}-10^{20} \mathrm{GeV}$ (Georgi, Quinn, and Weinberg, 1974), which is not so different from the boson effective masses needed to reduce the rate of proton decay sufficiently even when it occurs in lowest order. Of course there may exist a mechanism that prevents these boson masses from being arbitrarily large compared to those of the weak intermediate bosons (Gildener, 1976). We should discuss briefly suggestions that have been made for ways in which each of these mass scales might be reduced. We first discuss the mechanisms of proton decay and how its rate might be retarded. We then turn to the question concerning the unification mass scale.

In a theory with proton decay, there is no atomic mass number (or baryon number) $A$ that is exactly conserved. The case we have just mentioned, in which the proton decay proceeds in the lowest order of pertubation theory and the responsible bosons must have gigantic masses, is typically one in which there is no gauged quantum number $X$ that changes in proton decay and there is also no exactly conserved fermion number. The leptoquarks are also antidiquarks, and the decay proceeds directly in second order in $g^{2}$. If there is an exactly conserved fermion number, but still no gauged quantum number that is changed by proton decay, then the decay can be put off to a somewhat higher order of perturbation theory, but quite large boson masses are still needed. A typical example of this situation is provided by the group $E_{6}$, with the fermions put into 27 and $\overline{27}$ and with fermion number conserved; the decay amplitude is then of order $g^{4}$ rather than $g^{2}$.

Sufficiently slow proton decay without very large spin 1 boson masses is somewhat less difficult to achieve if we do have a generator $X$ of the group $G$ that must be broken (by an explicit Higgs mechanism or a hypothetical dynamical Higgs mechanism) in order for the decay to take place. Let us first consider the case in which fermion number is exactly conserved. In our discussion below of exact proton stability, we suppose that the Higgs violation, which gives a mass to the spin 1 boson gauging $X$, also breaks fermion number but preserves a linear combination, which is then $A$. Here we imagine that the Higgs violation leaves fermion number alone; $A$ can be defined in the same way but is no longer conserved. If we look at models with explicit Higgs bosons $\phi$ that accomplish this violation, we can assign them to various reducible representations of $G$ or else to a large irreducible one, in which some component has a nonzero vacuum expected value that gives a mass to the spin 1 boson gauging $X$ and at the same time induces, through virtual spin 1 bosons, an unrenormalizable effective coupling, with a finite calculable coefficient, that causes proton decay. Typically the parameters of the Higgs potential in $\phi$ space can be adjusted to make this decay very slow. (When renormalizable effective couplings are induced, then the coefficients are, in principle, arbitrary, and can only be estimated if we assume that a cutoff imitates whatever mechanism will eliminate the infinities in an improved theory.)

Pati and Salam $(1973,1974)$ have discussed this kind of slow violation of $A$ conservation, although their theory differs from the ones we consider in that their $G$ is not simple and their quarks are not confined and not fractionally charged. We can, however, as an example, replace their theory of four quark flavors and four lepton flavors by one in which the gauge group $G$ is $\mathrm{SU}_{16}$ and the spin $1 / 2$ fermions are put into 16 and $\overline{16}$, with conservation of fermions. The necessary Higgs violation to produce a slow proton decay can then be accomplished by $\phi$ fields in various reducible representations of $G$ or else by $\phi$ 's in an irreducible $(299,200$-dimensional!) representation. The $\phi$ component with nonzero expected value in the vacuum can then couple to three gauge bosons that convert three quarks into three leptons, ultimately inducing proton decay.

We have seen that the treatment of slow proton decay with a conserved fermion number resembles the discussion below of proton stability in which $A$ is defined as a linear combination of fermion number and $X$. We now consider the final case of retarded proton decay, where there is no fermion number but there is a generator $X$ that would prohibit the decay if it were exactly conserved, which it is not. That case resembles the treatment below of proton stability when there is no fermion number and $X$ coincides with $A$ in the spin $1 / 2$ sector of the theory. Here, instead of taking a conserved linear combination of $X$ and some quantity that is nonzero outside the spin $1 / 2$ sector, we just allow $X$ to equal $A$ and to be nonconserved. Again the dynamical or explicit Higgs bosons $\phi$ can be put into suitable representations of $G$ that allow them to give a mass to the spin-1 boson gauging $X$ and to induce the proton decay.

Although the coupling constants vary logarithmically with mass, the mass scale where the ratios of the electromagnetic and strong coupling constants become nearly equal to unity (that is, unification takes place) need not be anywhere near as large as the Planck mass. We give a brief review of the dependence of the unification mass on the theory. Let us first suppose that the symmetry violation occurs in only two stages, from $G$ to $\mathrm{SU}_{2} \times \mathrm{U}_{1} \times \mathrm{SU}_{3}^{c}$, and then to $\mathrm{U}_{1} \times \mathrm{SU}_{3}^{c}$. The unification mass may be computed from the renormalization group equations. Georgi, Quinn, and Weinberg (1975) have found that it is sensitive to the electric charge and weak isospin assignments made in the theory; in some examples it may be as low as $\sim 10^{9} \mathrm{GeV}$, but in others it is much larger than the Planck mass.

Particularly low unification masses can be achieved when the symmetry breakdown is a multistage process. This possibility has been considered by Fritzsch and Minkowski (1975), although their examples have the defect of violating quark-lepton universality in the early stages of the symmetry breakdown. We avoid that difficulty in the following example. Suppose that in the first stage $G$ breaks to $G_{A} \times G_{B}$, where the index of the adjoint representation of $G_{A}$ is much larger than that of $G_{B}$. (The index is the Casimir operator of the group for a given representation times the dimensionality of the representation divided by the number of generators. The coefficient occurring in the renormalization group equations is $11 / 3$ times the index of the adjoint minus $4 / 3$ times the sum of the indices of all Dirac fermion representations.) $G_{B}$ contains at least the minimal 
flavor group $\mathrm{SU}_{2} \times \mathrm{U}_{1}$ and $G_{A}$ contains $\mathrm{SU}_{3}^{c}$. The coupling constants for these factors $G_{A}$ and $G_{B}$ will vary at different rates as the renormalization mass further decreases, with the growth of the coupling of $G_{A}$ accelerated over that of $\mathrm{SU}_{3}^{c}$ alone. In due course $G_{A}$ breaks to $\mathrm{SU}_{3}^{c}$, but only after the coupling of $G_{A}$ has increased to a value much larger than the coupling for $G_{B}$. Thus, in a $2 n$-lepton, $2 n$-quark model based on $G=\mathrm{SU}_{8 n}$, the first stage of symmetry violation could break $\mathrm{SU}_{8 n}$ into $G_{B}=\mathrm{SU}_{n} \times \mathrm{SU}_{2} \times \mathrm{U}_{1}$ and $G_{A}=\mathrm{SU}_{3 n}$. At some smaller mass, the $\mathrm{SU}_{3 n}$ could break to $\mathrm{SU}_{3}^{c} \times \mathrm{SU}_{n}$. At GeV energies, the two $\mathrm{SU}_{n}$ groups could be inconspicuous, coupling light to heavy quarks and light to heavy leptons, leaving $\mathrm{SU}_{2} \times \mathrm{U}_{1} \times \mathrm{SU}_{3}^{c}$.

If the theory does contain mass scales of order of the Planck mass, then the unification procedure followed here, which omits gravitational processes, would be incomplete. It is sometimes suggested that it might be necessary to consider processes that appear to violate baryon number, such as the hypothetical radiation of all the energy of a small black hole by the Hawking (1975) effect. If quantum gravitation really permits such a process, then some kind of instability of the proton might be implied, but the whole matter is poorly understood. A more direct unification of Yang-Mills theory with Einsteinian gravitation is afforded by theories of extended supergravity, as discussed below.

Although there is no clear need for requiring an absolutely stable proton, one could try to impose exact proton stability and examine the consequences, pleasant or unpleasant. We do this here, ignoring the possibility that some kind of topological quantum number not apparent in the Lagrangian provides an atomic mass number. We examine the situations of dynamical symmetry breaking in some detail, and of explicit Higgs breaking briefly.

How may we obtain a conserved quantity $A$ in a unified gauge theory? Suppose the Lagrangian contains only spin $1 / 2$ and spin 1 fields, and the symmetry breakdown is dynamical. Then a single self-conjugate irreducible representation for the fermions precludes proton stability, since a conserved group generator $X$, if it survived the dynamical symmetry breakdown, would be associated with a massless vector boson and could not be used for A. Electric charge and color conservation are not enough to guarantee proton stability and we have already assumed there will be no accidental conservation laws. A pair of conjugate representations allows an ungauged conserved quantity $Z$ to be defined. (For complex representations, this doubling is required by CPT.) The quantity $Z$ cannot be $A$ since the quarks and leptons would all have the same value of $A$. However we can have $A$ conservation with $Z$ and $X$ (a local symmetry generator associated with a vector current) both broken and with some linear combination conserved. The boson becomes massive, and quarks and leptons can be distinguished. Since we do not explicitly calculate $A$ from the broken theory, our choice of the local generator $X$ presumes the appropriate symmetry-breaking patterns. If we had considered a reducible fermion representation with more complicated content, it would also be feasible to have, for example, exact lepton conservation. We do not analyze this possibility here. In any event, we as- sume that the eigenvalue spectrum of $A$ corresponds to a sensible atomic mass number.

The ordinary quarks must have $A=1 / 3$ so that the known baryons, which are composed of three of those quarks, all have $A=1$. Similarly the known leptons must have $A=0$. Then $A$ will be the usual baryon number for known matter. Unified theories also often predict other quarks and leptons. It is natural to require that their $A$ values'are constrained so color singlet states have integral values of $A$. Otherwise, we would need to accept the more drastic prediction of observable fractional $A$, including new stable particles corresponding to the lowest mass state for each allowed fractional value. Consequently, the $A$ value of a new quark falls in the sequence, $1 / 3$ plus integer, and new leptons have integral $A$. We call quarks with $A=\ldots,-5 / 3,-2 / 3,4 / 3,7 / 3, \ldots$. weird quarks, and leptons with $A= \pm 1, \pm 2, \ldots$ weird leptons. These weird fermions should be heavy enough to have escaped being observed, but otherwise pose no problems other than lack of economy. Weird baryons would contain at least one weird quark, and have inte$\operatorname{gral} A$, not equal to one. Weird mesons are $q \bar{q}$ states with $A$ a nonzero integer.

The exchange of bospns carrying color and flavor would be needed in the mechanism for producing a single weird particle. The color singlet bosons and the octet of gluons all carry $A=0$, but some of the bosons carry $1 / 3$ integral values of $A$. Two ordinary quarks could exchange such a boson, transforming one quark into a lepton with $A=0$ and the other quark into a weird antiquark with $A=2 / 3$, which could then combine with an ordinary quark to form a weird meson with $A=1$. If the boson masses are sufficiently large, this process would be suppressed. However, at sufficiently high energies pair production of weird particles would occur.

Weird particle decay does involve the exchange of a boson carrying $A=1 / 3$ and would be extremely slow if its mass were large. As examples, a weird meson with $A=1$ composed of a quark with $A=1 / 3$ and a weird antiquark with $A=2 / 3$ would have a lepton-baryon decay mode, and a weird baryon with $A=0$, composed of two quarks with $A=1 / 3$ and a weird quark with $A=-2 / 3$, might decay into three ordinary leptons, or into an ordinary meson and a lepton. We will be more specific in the examples.

With dynamical symmetry breaking there is only one case where proton stability does not imply weird particles: $G=\mathrm{SU}_{n}$ and the fermions in the n. But $A$ conservation may be imposed in any other gauge theory, as long as $f$ is large enough to include weird fermions. (In this regard $F_{4}$ and $E_{6}$ are flavor poor, if the fermions are assigned to only a few irreducible representations.) Table III lists the $A$ values that are implied by a simple choice of the local generator, as discussed in Sec.IV.

The situation of explicit Higgsism, although perhaps unattractive for nonsupersymmetric theories, does provide other solutions for the spectrum of $A$. Suppose the scalar and pseudoscalar fields imply new ungauged symmetries of the Lagrangian. As before $A$ can be constructed by taking the linear combination of a broken local and global generator that is conserved. For simplicity we restrict our attention to fermions in a single irreducible self-conjugate representation of $G$, so that 
TABLE III. Some examples of proton stabilization, with fermions doubled, and Majorana mass breaking. The general solutions are given in Sec. IV; these simple examples provide the most economical $A$ spectra.

\begin{tabular}{|c|c|c|c|c|}
\hline$G$ & Representation & (Equation) & $\mathrm{U}_{1}$ for $X$ & $A$ \\
\hline $\mathrm{SU}_{n}$ & $\mathbf{n}$ & $(2.4)$ & Explicit $U_{1}$ & $\frac{1}{3} N_{q}$ \\
\hline $\mathrm{SU}_{n}$ & $\left(\mathrm{n}^{k}\right)_{A}$ & $(2.11)$ & Explicit $U_{1}$ & $\frac{1}{3} N_{q}-\frac{2}{3} N_{r}+N_{L}$ \\
\hline $\mathrm{SO}_{n}$ & $\mathbf{n}$ & $(2.17)$ & $\begin{array}{l}\text { Flavor raid } \\
\left(\mathrm{SO}_{2 n^{\prime}} \supset \mathrm{SU}_{n^{\prime}} \times \mathrm{U}_{1}\right) \\
\text { plus explicit } \mathrm{U}_{1}\end{array}$ & $\frac{1}{3} N_{q}+\frac{4}{3} N_{r}+N_{L}$ \\
\hline $\mathrm{SO}_{n}$ & $\sigma$ & $\begin{array}{l}(2.22) \\
(2.24)\end{array}$ & Explicit $U_{1}$ & $\frac{1}{3} N_{Q}-\frac{2}{3} N_{r}+N_{L}$ \\
\hline$S p_{2 n}$ & $2 n$ & $\begin{array}{l}(2.25) \\
(2.26)\end{array}$ & $\begin{array}{l}\text { Flavor raid } \\
\left(S p_{2 n_{1}} \supset \mathrm{SU}_{n_{1}} \times \mathrm{U}_{1}\right) \\
\text { plus explicit } \mathrm{U}_{1}\end{array}$ & $\frac{1}{3} N_{q}+\frac{4}{3} N_{r}+N_{L}$ \\
\hline$E_{7}$ & 56 & $(2.36)$ & $\begin{array}{l}\text { Flavor raid } \\
\mathrm{SU}_{6} \supset \mathrm{SU}_{5} \times \mathrm{U}_{1}\end{array}$ & $\frac{1}{3} N_{q}+\frac{1}{3} N_{\sigma}+\frac{4}{3} N_{s}-\frac{2}{3} N_{r}+N_{L}$ \\
\hline
\end{tabular}

the global generator $Z$ is zero for the fermions. Then the local generator alone is $A$ for the fermions.

The phenomenology of weird particle production and decay is similar to before, except that the exchange of scalar particles that carry both flavor and color should be included. Weird particles can be completely avoided in the $\mathrm{n}$ of $\mathrm{SO}_{n}$ and the $2 \mathrm{n}$ of $S p_{2 n}$, but otherwise some weirdness is also needed in this symmetry-breaking scheme. Again, the details are worked out in Sec.IV.

The embedding of $\mathrm{SU}_{3}^{c}$, electromagnetic $\mathrm{U}_{1}$, and the local $U_{1}$ for stabilizing the proton are all easily extended to $G \times G$ theories. Color can be embedded in $G \times G$ in essentially two ways.

$\mathrm{SU}_{3}^{c}$ may be explicitly contained in just one $G$ factor, and the two $G$ factors are transformed into one another by some abstract reflection principle. The $\mathrm{SU}_{4} \times \mathrm{SU}_{4}$ model of Pati and Salam (1974) is an example. The fermion representations are of the form, $\left(\mathbf{r}_{1}, \mathbf{r}_{2}\right)$. If the second $G$ factor contains $\mathrm{SU}_{3}^{c}$, then $\mathrm{r}_{2}$ must be one of the representations in Table $I$, and there is no restriction on $r_{1}$. Such models are similar in spirit to the ones already discussed, so we do not consider them further.

The other possibility, to be considered in more detail, is that $\mathrm{SU}_{3}^{c}$ is generated by the sum of generators of $\mathrm{SU}_{3}$ subgroups of each $G$ factor. The color content of a $G \times G$ representation $\left(\mathbf{r}_{1}, \mathbf{r}_{2}\right)$ then contains the color representations in $r_{1} \times r_{2}$. The restriction that $\left(r_{1}, r_{2}\right)$ have at most $1^{c}, 3^{c}$, and $\overline{3}^{c}$ then implies that $r_{1}$ or $r_{2}$ must be an $\mathrm{SU}_{3}$ singlet. This in turn imposes the requirement that $\mathbf{r}_{1}$ be a $G$ singlet and $\mathbf{r}_{2}$ be one of the representations in Table I, or vice versa. The only possible fermion representations are then linear combinations of $\left(1, \mathbf{r}_{2}\right)$ and $\left(r_{1}, 1\right)$.

We first consider the chiral structure of the fermion representation for the case where the reflection symmetry between the two $G$ factors is not parity. If $f_{L}$ has the form $\left(r_{1}, r_{2}\right)$, then $f_{R}$ transforms as $\left(r_{1}, r_{2}\right)$ or $\left(\bar{r}_{1}, \bar{r}_{2}\right)$. We simply obtain the vectorlike and flavor chiral theories, respectively, already summarized in Table II. In this case there is an $\mathrm{SU}_{3} \times \mathrm{SU}_{3}$ of vector currents and there is the temptation of enlarging $\mathrm{SU}_{3}^{c}$ to $\mathrm{SU}_{3} \times \mathrm{SU}_{3}$. Although this way of enlarging the color group differs from Case 3 in Sec.II, that discussion is still relevant. Otherwise, these $G \times G$ theories are similar to the $G$ simple theories already discussed.

Finally, we may suppose that parity is the reflection symmetry between the $G$ factors, although this possibility raises several questions. If $f_{L}$ is $\left(r_{1}, r_{2}\right), f_{R}$ must be $\left(\mathbf{r}_{2}, \mathrm{r}_{1}\right)$ or $\left(\overline{\mathbf{r}}_{2}, \overline{\mathbf{r}}_{1}\right)$ : the first case is chiral, and the second case is conjugate chiral. Both $r_{1}$ and $r_{2}$ must be safe representations of $G$ for the triangle anomalies to be absent, but safety through an accidental cancellation is a possibility as above. For simplicity we still ignore Class II theories in this connection.

The strong gauge group in these $G \times G$ theories is a chiral $\mathrm{SU}_{3} \times \mathrm{SU}_{3}$. There are several objections to considering such theories. First, the chiral quark-gluon theory by itself has anomalous divergences, which are cut off only at a very large energy by the unified theory. As always, we assume that the unbroken $\mathrm{SU}_{3}^{c}$ is generated by the sum of the corresponding $\mathrm{SU}_{3}$ generators. The axial symmetries, generated by the differences, must be broken, and we may characterize the violation by a kind of effective mass-squared $m_{A}^{2}$ for the axial gluons inside the hadrons. This parameter $m_{A}^{2}$ must be at least several $(\mathrm{GeV})^{2}$ in order to avoid quark-quark spin-spin forces of the wrong sign and other unsuitable forces among quarks and antiquarks. When flavor radiative corrections are put in, there may also be parity-violating amplitudes of order $\alpha_{\text {strong }} \alpha_{\text {weak }} m_{A}^{-2}$ that require $m_{A}^{2}$ to be even larger. For all these reasons, we shall not pay any more attention to chiral $G \times G$ theories.

The enumeration of possible charge assignments for $G \times G$ theories is identical to that for the $G$-simple theories, since $r_{1}$ or $r_{2}$ must be a $G$ singlet. The electric charge operator is simply the sum of generators of two corresponding $U_{1}$ 's from the $G^{11}$. subgroups of each $G$ factor. The same argument holds for extracting $X$ for stabilizing the proton. Thus we restrict our considerations to simple gauge groups, while recognizing that the results are valid for $G \times G$ theories.

Although some of the theories included in our classification are quite attractive, we find none so appealing that it should blind us to promising lines of development 
that have been excluded here. We consider briefly two of the more popular directions of research: (1) enlarging the global symmetry structure of the Lagrangian; (2) enlarging the local symmetry structure. In both cases, the Lie group symmetry of the Lagrangian is extended to a "graded" or "super" symmetry. The supersymmetry operators, which are adjoined to the Lie algebra, satisfy anticommutation relations with one another, and commutation relations with the members of the Lie algebra. They transform bosons into fermions, so that ir reducible supermultiplets must contain both.

We first consider ordinary global supersymmetry (Golfand \& Likhtman, 1971; Wess and Zumino, 1974) in direct product with a Yang-Mills internal symmetry (Salam and Strathdee, 1975; Ferrara and Zumino, 1974). The adjoint representation of gauge bosons is then accompanied by an adjoint representation of Majorana spin $1 / 2$ fermions, which must include $8^{c}$. Once color octet fermions are required, it is not so natural to demand that the fundamental representation of $G$ be restricted to $1^{c}, 3^{c}$, and $\overline{3}^{c}$ only. However, in any new embeddings, the flavor singlet set of generators would be larger than one or two 8's; thus there would be many new possibilities for the color group, and there would also be a further proliferation of fermions with new color representations. We conclude, therefore, that a globally supersymmetric theory with internal symmetry treated by means of a direct product would probably involve only embeddings treated in this article; the principal change would be to include a set of spin 1/2 Majorana fermions belonging to the adjoint representation, which is fully treated here for the description of the spin 1 bosons.

If the only supermultiplet considered is the one with spin 1 and spin $1 / 2$ belonging to the adjoint representation of $G$, then the symmetry breakdown must be dynamical. However, it is not so ugly to introduce a supermultiplet of matter fields consisting of Majorana fermions, scalars, and pseudoscalars transforming as some representation of $G$, which could well be the adjoint and/ or one of the representations discussed here for the spin $1 / 2$ fermions. In that case, the Lagrangian is available for inspection, and in reality it is hard to break both the supersymmetry degeneracies and the internal symmetry degeneracies simultaneously as needed (Fayet and Iliopoulos, 1974). If these schemes do work, there will be a Goldstone fermion, which is not the neutrino since it decouples in the zero frequency limit (Freedman and de Wit, 1975; Bardeen, 1975).

The direct product is not the only way to combine "internal" symmetry with supersymmetry. We can have $N$ fold extended supersymmetry (Salam and Strathdee, 1974 ) in which there are $N$ supersymmetry operators belonging to the vector representation of the "internal" group $\mathrm{SO}_{N}$ or $\mathrm{SU}_{N}$. The supermultiplets then contain particles with more and more different spin values as $N$ increases. If the group is made large enough to be the gauge group $G$ of a unified Yang-Mills theory, then one is led to spins of $3 / 2$ and higher and severe difficulties are then encountered in a theory with purely global extended supersymmetry.

We may consider, however, a case in which $N$ is not very large and we do not attempt to gauge the $\mathrm{SO}_{N}$ or $\mathrm{SU}_{N}$ symmetry of extended supersymmetry by means of a
Yang-Mills theory. Rather, we consider a direct product of extended supersymmetry and a Yang-Mills symmetry group $G$. Let us take the largest value of $N$ that allows us to avoid spin $3 / 2$ particles in the supermultiplet containing the Yang-Mills fields, namely $N=4$ (Fayet, 1976; Brink, Schwarz, and Scherk, 1977). One then has a set of vector particles in the adjoint representation of $G$, as well as four sets of Majorana spin $1 / 2$ particles, and three sets each of scalar and pseudoscalar particles, also in the adjoint representation. For such a case, as for the direct product of $G$ with ordinary supersymmetry, our embeddings may well be sufficient and the adjoint representation, described in our work for the spin 1 bosons, can be used also for the spin $1 / 2$ fermions and the spin 0 bosons, in the requisite number of copies. The renormalization group for this type of theory has the fascinating property that the leading $g^{4}$ term in the GellMann-Low function $\psi\left(g^{2}\right)$ vanishes (Ferrara, 1976). It has been remarked (Abbott et al., 1977; Curtright, 1977) that as a consequence the leading term in the anomalous divergence of the supercurrent (ignoring supergravity) also vanishes. Very recently (Jones, 1977, and Poggio and Pendleton, 1977) calculations of $\psi\left(g^{2}\right)$ have been extended to order $g^{6}$ using the method of Jones (1975) and it has been shown that the scale covariance $\psi\left(g^{2}\right)=0$ persists to that order, a fascinating result of unknown significance.

We shall return to this class of theories below, merely remarking here that they have a global $\mathrm{SU}_{4} \approx \mathrm{SO}_{6} \mathrm{sym}-$ metry, and if such theories are to be useful, then we must learn how that symmetry can be spontaneously violated without contradicting experience.

Now let us discuss the treatment of gravitation in supersymmetry theory. The graviton must belong to a supermultiplet and one is immediately led to local supersymmetry or supergravity (Freedman, van Nieuwenhuizen, and Ferrara, 1976; Deser and Zumino, 1976) in which the graviton, which gauges the Poincare group, is accompanied by one or more spin $3 / 2$ particles that gauge supersymmetry or extended supersymmetry. (These massless spin $3 / 2$ particles may eat the Goldstone fermions and become massive.) Plain $N=1$ supergravity has just a single spin $3 / 2$ particle forming a supermultiplet with the graviton and is compatible with the introduction of direct-product internal symmetry as described above, with spin 1 and spin $1 / 2$ particles in the adjoint representation of some group $G$ and possibly spin $1 / 2$, scalar, and pseudoscalar particles in some representation of $G$. However, such a theory is not renormalizable. (In any event, the applicability of our work would be the same as discussed above for the use of global supersymmetry.)

The most interesting type of theory so far proposed is probably extended supergravity (with $2 \leqslant N \leqslant 8$ ), especially the version with $N=8$, in which a single spin 2 graviton is accompanied by an $\mathrm{SO}_{8}$ octet of spin $3 / 2$ particles, a set of spin 1 particles belonging to the 28-dimensional adjoint representation of $\mathrm{SO}_{8}$, a set of Majorana spin $1 / 2$ particles belonging to the 56 of $\mathrm{SO}_{8}$, and scalar and pseudoscalar multiplets in two 35-dimensional representations of $\mathrm{SO}_{8}$.

The coupling involving Newton's constant is presumably supplemented by a dimensionless coupling that in- 
cludes Yang-Mills behavior of the spin 1 bosons, which would thus gauge the group $\mathrm{SO}_{8}$. The dimensionless coupling induces a cosmological term in Einstein's gravitational equation that is much too large; that is a difficulty unless some spontaneous violation of symmetry permits the cosmological term to be canceled almost completely. But the major problem with $\mathrm{SO}_{8}$ supergravity is that $\mathrm{SO}_{8}$ is too small a group to include color $\mathrm{SU}_{3}$ times a sufficiently large flavor group as a subgroup.

One may speculate about a future unified field theory of all interactions and all elementary particles that would resemble $\mathrm{SO}_{8}$ supergravity but involve sacrificing some principle now held sacred, so that the notion of extended supergravity could be generalized. In such a hypothetical theory, an internal symmetry group $G$ larger than $\mathrm{SO}_{8}$ would be gauged by spin 1 bosons, and both the spin $3 / 2$ and spin $1 / 2$ fermions would be assigned to representations of $G$. It is then very natural to suppose that the spin $3 / 2$ fermions would belong to some basic representation of $G$ and would include only color singlets, triplets, and antitriplets. In that case our embeddings of $\mathrm{SU}_{3}^{c}$ in $G$ would be exhaustive and the representations that we study in this article for assignment to spin $1 / 2$ fermions would be precisely relevant for the spin $3 / 2$ fermions. (The spin $1 / 2$ particles would presumably be assigned to more complicated representations.) The possibility that our review might be used in this way is an exciting one, although highly speculative, and has encouraged us to prepare it for publication after some years of delay.

Another fascinating possibility for a unified theory involves the direct product of $N=4$ extended supersymmetry with a gauged internal-symmetry group $G$, as described above in the global case. We introduce local $N$ $=4$ supersymmetry; the graviton then belongs to a supermultiplet along with four spin $3 / 2$ particles, six vector particles, four Majorana spin 1/2 particles, a scalar, and a pseudoscalar. This supermultiplet is coupled to itself with Newton's constant. The generalized YangMills "matter field" discussed above is then introduced, containing one vector particle, four Majorana spin $1 / 2$ particles, three scalars, and three pseudoscalars for each component of the adjoint representation of $G$. This "matter field" is then coupled to the gravity supermultiplet by Newton's constant and to itself by a dimensionless charge. The resulting theory is then no doubt unrenormalizable (like the $N=1$ supergravity theory with added matter multiplets mentioned earlier). However, a theory like the one we are discussing, at least for $G$ $=\mathrm{SU}_{n}$ for any $n$, can be obtained as an approximation, for energies small compared to the Planck mass, to the remarkable ten-dimensional string theory of Scherk and Schwarz $(1974,1975)$, in which six dimensions are "compactified" into a tiny ball so as to be physically insignificant at any reasonable energy, leaving effectively a fourdimensional theory. [For the original theory in 10 actual dimensions, see Ramond (1971) and Neveu and Schwarz (1971).] All elementary particles lie on Regge trajectories with a slope $\alpha^{\prime}$ of the order of Newton's constant for the closed strings (giving the gravitational supermultiplet) and a slope $2 \alpha^{\prime}$ for the open strings (giving the particles of the matter field). The string version of the theory has been shown (Gliozzi et al., 1977) to be free of ghosts and tachyons and may well be renormalizable. It does not have the difficulty of introducing a cosmological term into Einstein's gravitational equation, nor does it have to make use of an internal group $G$ that is too small. Given $G$, our embeddings are probably the relevant ones, and we must utilize the adjoint representation, listed here for the spin 1 bosons, also for the spin 1/2 Majorana fermions (four times) and for the scalar and pseudoscalar bosons (three times each).

We conclude this introduction with some examples of unified models. Our main purpose is to illustrate the color embeddings, charge assignments, and the definition of an atomic mass number; the detailed phenomenology of many models is available in the literature and not reviewed here. There are five examples corresponding to Cases 1, 4, 2, 5, and 9, respectively (see Table I). In our examples we examine in some more detail the implications of the possible requirement that the proton be absolutely stable. Although we assume the gauge group is broken down to $\mathrm{U}_{1}^{e m} \times \mathrm{SU}_{3}^{c}$, we look only at the details of the symmetry breaking needed for proton stabilization. We should emphasize that Secs.II and III also apply to proton unstable theories, and models like the one based on $E_{6}$ should not be discarded simply because the proton stable version must be fairly complicated. Much that we say here is summarized in Tables IV-VIII.

As a first example, we briefly discuss the familiar $\mathrm{SU}_{n}$ models, where the flavor group is identified in Eq. $(2.7)$, the vector bosons are listed in Eq. (2.6), and the $n_{1}$ leptons and $n_{3}$ quarks are assigned to $\mathrm{n}$, with $n=n_{1}+3 n_{3}$ in Eq. (2.4). The antifermions are assigned to $\bar{n}$, and the Lagrangian has an additional global symmetry generated by fermion number. Since the $\mathrm{n}$ is a complex representation of $\mathrm{SU}_{n}$, this theory must be vectorlike to avoid triangle anomalies; see, for example, Fritzsch, GellMann, and Minkowski, 1975; Kingsley, Treiman, Wilczek, and Zee, 1975; de Rújula, Georgi, and Glashow, 1975; and Pakvasa, Simmons, and Tuan, 1975.

It is commonly assumed that quarks and leptons occur in $\mathrm{SU}_{2}$ doublets with the standard charge assignment. Then for each lepton doublet with charges 0 and -1 , there must be a quark doublet with charges $2 / 3$ and $-1 / 3$ for the sum of the fermion charges to be zero. This version of the $\mathrm{SU}_{n}$ model has a purely vectorial weak neutral current. Of course many other $\mathrm{SU}_{2}$ (and even charge) assignments are possible and may agree better with experiment.

Most of the physical content of this theory is bound up in the symmetry breakdown; not even the universality of the weak and electromagnetic couplings of quarks and leptons is present in the unbroken Lagrangian, since different sets of flavor bosons couple to quarks and to leptons. (See Table IV.) The proton is stable in the unbroken theory because of the conservation of the explicit $U_{1}$ in $G^{11}$ and of fermion number. But the explicit $U_{1}$ must be broken to avoid an unwanted massless boson. If we assume that the broken theory conserves only electric charge, fermion number and color, then the process $q q q \rightarrow l l l$ is allowed.

There is no reason a priori that the symmetry violation should not break fermion number. For example, a Majorana neutral lepton mass term breaks both fermion number and the explicit $U_{1}$ in $G^{f 1}$, but does not break the 
TABLE IV. Boson-fermion couplings in $\mathrm{SU}_{n}$ models with fermions in $\mathbf{n}$. The table lists the vector bosons in the unbroken theory that provide the $a \rightarrow b+$ boson transition, $a$ and $b$ are fermions. See Eqs. (2.4) and (2.6). The $A$ values for the proton-stabilizing symmetry breaking discussed in the text are also given.

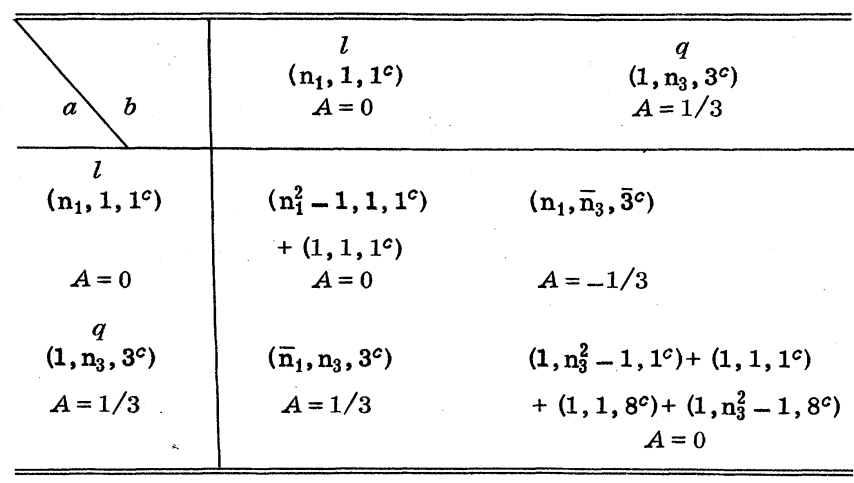

linear combination of the generators, $A=N_{\alpha} / 3$, which implies that $A$ is conserved. The leptoquarks, assigned to $\left(\overline{\mathbf{n}}_{1}, \mathrm{n}_{3}, 3^{c}\right)$ of Eq. (2.6), then carry $A=1 / 3$. The symmetry-breaking terms needed for $q q q \rightarrow l l l$ must all vanish in this case, since they would carry nonzero values of $A$. This specific class of $\mathrm{SU}_{n}$ models is summarized in Table IV.

Our second example involves several choices. We assign the fermions to the $\mathrm{n}$ of $\mathrm{SO}_{n}$, which is a self-conjugate representation, and the theory is vectorlike. Suppose that all fermions are assigned to a single $\mathbf{n}, \mathrm{Eq}$. (2.17), that there is no additional global $U_{1}$, and that the explicit local $U_{1}$ is broken. As long as electric charge and color are the only conserved quantities, the $3^{c}$ boson-mass eigenstates will be mixtures of the $\left(n_{1}, n_{3}, 3^{c}\right)$ and $\left[1, \overline{\frac{1}{2} \mathrm{n}_{3}\left(\mathrm{n}_{3}+1\right)}, 3^{c}\right]$ bosons in Eq. (2.18). Then the leptoquark is also an antidiquark, and its exchange can, in general, cause the transition $q q q \rightarrow q \bar{q} l$, so that the pro- ton is unstable in second order.

If we want to stabilize the proton, we must decide whether to obtain the global from some other sector of the theory or to double the fermion representation, thereby providing a fermion number. In the former case it is possible to obtain a scheme with no weird particles; see the discussion around Eq. (4.28). In the latter, the generator of the relevant local $\mathrm{U}_{1}$ must have a nonzero value for leptons, which requires going beyond the explicit $\mathrm{U}_{1}$. For simplicity we limit this example to $n$ even and extract a second $\mathrm{U}_{1}$ from $\mathrm{SO}_{n 1} \supset \mathrm{SU}_{n^{*}} \times \mathrm{U}_{1}$ where $n^{\prime}$ $=n_{1} / 2$. We refer to the procedure of taking part of the $U_{1}$ from a non-Abelian factor of $G^{\mathrm{f} 1}$ as a "flavor raid." The local $U_{1}$, generated by the sum of this $U_{1}$ generator and the explicit $U_{1}$ generator, will be broken together with fermion number [see Eq. (4.12) and the discussion beneath]. The most economical scheme as far as weirdness is concerned has leptons with $A=0$ and 1 , and quarks with $A=1 / 3$ and $-2 / 3$. The $A$ values of the vector bosons are easily worked out from Eqs. (2.17) and (2.18), as is the phenomenology of weird decays. (The latter is similar to some examples worked out below.) This scheme is summarized in Table $\mathrm{V}$.

As a third example, let us look at a Class III SU mod- $_{n}$ el (Case 2). For this example, we give a simple discussion of the phenomenology of weird fermions. The summary of this model for arbitrary $n$ is contained in Table VI, but for definiteness we analyze a vectorlike $\mathrm{SU}_{8}$ model where the fermions are assigned to the $56=\left(8^{3}\right)_{A}$, which is complex. The flavor group is $\mathrm{SU}_{5} \times \mathrm{U}_{1}, \mathrm{Eq}$. (2.10), and the Lagrangian possesses a global $U_{1}$ symmetry, which is generated by fermion number. The $\mathrm{SU}_{5}$ $\times \mathrm{SU}_{3}^{c}$ decomposition, Eq. (2.11), of the 56 of fermions is

$$
56=\left(10,1^{c}\right)+\left(10,3^{c}\right)+\left(5, \overline{3}^{c}\right)+\left(1,1^{c}\right),
$$

while the vector bosons are assigned to the adjoint 63 , Eq. (2.9)

$$
63=\left(24,1^{c}\right)+\left(1,1^{c}\right)+\left(1,8^{c}\right)+\left(\overline{5}, 3^{c}\right)+\left(5, \overline{3}^{c}\right) .
$$

TABLE V. Boson-fermion couplings in the $\mathrm{SO}_{2 n}$ model. Representations are decomposed under $\mathrm{SO}_{2 n} \supset \mathrm{SU}_{n} \times \mathrm{U}_{1} \times \mathrm{SU}_{n_{3}} \times \mathrm{U}_{1} \times \mathrm{SU}_{3}^{c}$,

\begin{tabular}{|c|c|c|c|c|}
\hline$a \backslash b$ & $\begin{array}{c}\bar{l} \\
\left(\overline{\mathrm{n}}^{\prime}, 1,1^{c}\right) \\
A=0\end{array}$ & $\begin{array}{c}q \\
\left(1, \mathrm{n}_{3}, 3^{c}\right) \\
A=1 / 3\end{array}$ & $\begin{array}{c}\bar{r} \\
\left(1, \overline{\mathrm{n}}_{3}, \overline{3}^{c}\right) \\
A=2 / 3\end{array}$ & $\begin{array}{c}L \\
\left(\mathrm{n}^{\prime}, 1,1^{c}\right) \\
A=1\end{array}$ \\
\hline $\begin{array}{l}\quad \bar{l} \\
\left(\overline{\mathbf{n}^{\prime}}, 1,1^{c}\right) \\
A=0\end{array}$ & $\begin{array}{l}\left(\mathrm{n}^{\prime 2}-1,1,1^{c}\right)+2\left(1,1,1^{c}\right) \\
A=0\end{array}$ & $\begin{array}{l}\left(\overline{\mathbf{n}}^{\prime}, \overline{\mathrm{n}}, \overline{3}^{c}\right) \\
A=-1 / 3\end{array}$ & $\begin{array}{l}\left(\overline{\mathbf{n}}^{\prime}, \overline{\mathbf{n}}_{\mathbf{3}}, 3^{c}\right) \\
A=-2 / 3\end{array}$ & $\begin{array}{l}\left.\overline{\left[\frac{1}{2} \mathrm{n}^{\prime}\left(\mathrm{n}^{\prime}-1\right)\right.}, 1,1^{c}\right] \\
A=-1\end{array}$ \\
\hline $\begin{array}{c}q \\
\left(1, \mathrm{n}_{3}, 3^{c}\right)\end{array}$ & $\left(\mathrm{n}^{\prime}, \overline{\mathrm{n}}_{3}, 3^{c}\right)$ & $\left(1, \mathrm{n}_{3}{ }^{2}-1,1^{c}\right)+\left(1,1,1^{c}\right)$ & {$\left[1, \frac{1}{2} n_{3}\left(n_{3}+1\right), \overline{3}^{c}\right]$} & $\left(\overline{\mathbf{n}}^{\prime}, \mathrm{n}_{3}, 3^{c}\right)$ \\
\hline$A=1 / 3$ & $A=1 / 3$ & $\begin{array}{l}+\left(1,1,8^{c}\right) \\
A=0\end{array}$ & $\begin{array}{l}+\left[1, \frac{1}{2} \mathrm{n}_{3}\left(\mathrm{n}_{3}-1\right), 6^{c}\right] \\
A=-1 / 3\end{array}$ & $A=-2 / 3$ \\
\hline$\left(\overline{1}, \overline{\mathbf{n}}_{3}, \overline{3}^{c}\right)$ & $\left(\mathrm{n}^{\prime}, \overline{\mathbf{n}}_{3}, \overline{3}^{c}\right)$ & {$\left[1, \overline{\frac{1}{2} \mathrm{n}_{3}\left(\mathrm{n}_{3}+1\right)}, 3^{c}\right]$} & $\left(1, \mathrm{n}_{3}^{2}-1,1^{c}\right)$ & $\left(\overline{\mathbf{n}}^{\prime}, \overline{\mathbf{n}}_{3}, \overline{\mathbf{3}}^{c}\right)$ \\
\hline$A=2 / 3$ & $A=2 / 3$ & $\begin{array}{l}+\left[1, \overline{\frac{1}{2} \mathrm{n}_{3}\left(\mathrm{n}_{3}-1\right)}, \overline{6}^{c}\right] \\
A=1 / 3\end{array}$ & $\begin{array}{l}+\left(1,1,1^{c}\right)+\left(1,1,8^{c}\right) \\
A=0\end{array}$ & $A=-1 / 3$ \\
\hline $\begin{array}{c}L \\
\left(n^{\prime}, 1,1^{c}\right)\end{array}$ & {$\left[\frac{1}{2} n^{\prime}\left(n^{\prime}-1\right), 1,1^{c}\right]$} & $\left(\mathbf{n}^{\prime}, \overline{\mathbf{n}}_{3}, \overline{3}^{c}\right)$ & $\left(\mathbf{n}^{\prime}, \mathrm{n}_{3}, 3^{c}\right)$ & $\left(n^{\prime 2}-1,1,1^{c}\right)$ \\
\hline$A=1$ & $A=1$ & $A=2 / 3$ & $A=1 / 3$ & $\begin{array}{l}+2\left(1,1,1^{c}\right) \\
A=0\end{array}$ \\
\hline
\end{tabular}
$n=n^{\prime}+3 n_{3}$. The local generator used in defining $A$ is a linear combination of the two $U_{1}$ 's explicit in this decomposition. 
TABLE VI. Boson-fermion couplings in Class $\mathrm{III} \mathrm{SU}_{n}$ models. The $A$ assignments are made assuming that $X$ generates the explicit $\mathrm{U}_{1}$ in Eq. (2.10).

\begin{tabular}{|c|c|c|c|c|}
\hline$a \backslash b$ & $\begin{array}{c}\bar{l} \\
\left(\left(\mathrm{n}_{1}^{k}\right)_{A}, 1^{c}\right) \\
A=0\end{array}$ & 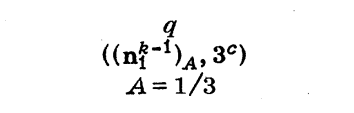 & $\begin{array}{c}\bar{r} \\
\left(\left(\mathbf{n}_{1}^{k-2}\right)_{A}, \overline{3}^{c}\right) \\
A=2 / 3\end{array}$ & $\begin{array}{c}L \\
\left(\left(\mathrm{n}_{1}^{k-3}\right)_{A}, 1^{c}\right) \\
A=1\end{array}$ \\
\hline $\begin{array}{l}\quad \bar{l} \\
{\left[\left(\mathrm{n}_{1}^{k}\right)_{A}, 1^{c}\right]} \\
A=0\end{array}$ & $\begin{array}{l}\left(\mathrm{n}_{\mathbf{1}}^{2}-1,1^{c}\right)+\left(1,1^{c}\right) \\
A=0\end{array}$ & $\begin{array}{l}\left(\mathrm{n}_{1}, \overline{3}^{c}\right) \\
A=-1 / 3\end{array}$ & None & None \\
\hline $\begin{array}{l}\stackrel{q}{q}\left[\left(\mathbf{n}_{1}^{k-1}\right)_{A}, 3^{c}\right] \\
A=1 / 3\end{array}$ & $\begin{array}{l}\left(\overline{\mathrm{n}}_{1}, 3^{c}\right) \\
A=1 / 3\end{array}$ & $\begin{array}{l}\left(n_{1}^{2}-1,1^{c}\right)+\left(1,1^{c}\right)+\left(1,8^{c}\right) \\
A=0\end{array}$ & $\begin{array}{l}\left(\mathrm{n}_{1}, \overline{3}^{c}\right) \\
A=-1 / 3\end{array}$ & None \\
\hline $\begin{array}{l}\quad \bar{r} \\
{\left[\left(\mathbf{n}_{1}^{k-2}\right)_{A}, \overline{3}^{c}\right]} \\
A=2 / 3\end{array}$ & None & $\begin{array}{l}\left(\overline{\mathbf{n}}_{1}, 3^{c}\right) \\
A=1 / 3\end{array}$ & $\begin{array}{l}\left(\mathrm{n}_{1}^{2}-1,1^{c}\right)+\left(1,1^{c}\right)+\left(1,8^{c}\right) \\
A=0\end{array}$ & $\begin{array}{l}\left(\mathrm{n}_{1}, \overline{3}^{c}\right) \\
A=-1 / 3\end{array}$ \\
\hline $\begin{array}{l}\stackrel{L}{\left[\left(\mathrm{n}_{1}^{k-3}\right)_{A}, 1^{c}\right]} \\
A=1\end{array}$ & None & None & $\begin{array}{l}\left(\overline{\mathbf{n}}_{1}, 3^{c}\right) \\
A=1 / 3\end{array}$ & $\begin{array}{l}\left(1,1^{c}\right) \\
A=0\end{array}$ \\
\hline
\end{tabular}

There are 25 flavor bosons, including the photon, and three known weak bosons. The other bosons, which transform as $\left(\overline{5}, 3^{c}\right)$ and $\left(5, \overline{3}^{c}\right)$, mediate transitions between $\bar{l}$ in $\left(\overline{10}, 1^{c}\right)$ and $q$ in $\left(10,3^{c}\right)$, between $q$ and $\bar{\gamma}$ in $\left(5, \overline{3}^{c}\right)$, and between $\bar{r}$ and the singlet " $L$ " lepton. (See Table VI.)

The electric charge assignments are easily cataloged from Eqs. (3.3) and (3.4). It is rather natural to choose the standard charges since it is the only assignment in which the charge spread of the l's and q's is less than 4, the lepton charges are less than 3 , and there is more than one neutral lepton. Then $\operatorname{six} l$ leptons have $Q=-1$ and the remaining four have $Q=0$; four of the $q$ quarks have $Q=2 / 3$, and six have $Q=-1 / 3$; four $r$ quarks have $Q=2 / 3$ and the other one has $Q=-1 / 3$; and the $L$ lepton has $Q=-1$. Note that the $L$ and $\bar{l}$ leptons cannot be mixed by symmetry breaking, since they have no charges in common.

If there is an $\bar{r} q$ meson less massive than the proton, then the proton may decay in second order into this meson plus a lepton. If the $L$ is light enough, the proton can decay into three leptons, $\overline{l l} L$. But if the light quarks are all of $q$-type, and the light leptons are of $l$-type, then it is perhaps natural to suppose that the $(\bar{r} q)$ mesons and also $L$ leptons would be heavier than the proton. In this case, the proton is guaranteed to be stable until the explicit $U_{1}$ of Eq. (2.10) is broken. Moreover, if fermion number is not finally broken, the proton decay to three light leptons does not violate the remaining conservation laws, although this process could be very slow.

The proton can, of course, be stabilized by breaking fermion number together with a $U_{1}$ in $G^{\mathrm{f} 1}$. The simplest solution uses the explicit $U_{1}$; the general solution is given in Eqs. (4.11) and (4.12). We avoid making a flavor raid in this example, so that the $\bar{l}$ leptons then have $A=0$; the $q$ quarks have $A=1 / 3$, the $r$ quarks have $A$ $=-2 / 3$; and the $L$ lepton has $A=1$. [See Eqs. (4.7)(4.10).] The model, $A$ assignments, and boson couplings are summarized in Table VI.

In order to effect transitions from weird to ordinary fermions, a boson carrying $A$ must be involved. The bosons in the $\left(\overline{5}, 3^{c}\right)$ carry $A=1 / 3$, those in the $\left(5, \overline{3}^{c}\right)$ carry
$A=-1 / 3$, and the remaining bosons have $A=0$. The $\left(\overline{5}, 3^{c}\right)$ bosons mediate the transition $r \rightarrow \overline{l q q}$ in second order. Thus a weird baryon with $A=0$ composed of $r q q$ could decay into a lepton and two mesons. Similarly a weird meson with $A=1$ composed of $\bar{r} q$ would decay into a baryon and a lepton. The only second-order transitions from $L$ into other fermions are $L \rightarrow \overline{r v q}$ and $L \rightarrow \bar{r} q l$, so the $L$ decay may be a two-step process. $L$ 's produced in pairs in $e^{+} e^{-}$annihilation could be nearly stable.

Our fourth example is again a model with a Class III embedding, but the theory may be either vectorlike or flavor chiral. We assign the fermions to the spinor representation of $\mathrm{SO}_{14}$, with color embedded as $\mathrm{SO}_{14} \supset \mathrm{SO}_{8}$ $\times \mathrm{U}_{1} \times \mathrm{SU}_{3}^{c}$. Models of this kind have been studied by Fritzsch and Minkowski, 1975. The $\mathrm{SO}_{14}$ spinor is complex and 64-dimensional, and in the vectorlike model the quark and lepton content is given by Eq. (2.24)

$$
64=\left(8,1^{c}\right)+\left(8,3^{c}\right)+\left(8^{\prime}, 1^{c}\right)+\left(8^{\prime}, \overline{3}^{c}\right),
$$

where 8 and $8^{\prime}$ are the two real inequivalent spinor representations of $\mathrm{SO}_{8}$. The antifermions are, of course, assigned to the $\overline{64}$. Thus all the left-handed spin $1 / 2$ states are clássified by $64+\overline{64}$, as are the right-handed states in the vectorlike model.

Among the solutions for the electric charge assignments given in Eq. (3.5)-(3.8), there are two interesting ones: the standard charge assignment is recovered with $n_{1}=1$ and $n_{2}=n_{3}=n_{4}=0$. Half of the leptons have $Q=-1$, and the other half have $Q=0$; half the quarks have $Q=2 / 3$, the other half have $Q=-1 / 3$. For the solution, $n_{1}=n_{2}=n_{3}=1$ and $n_{4}=0$, the leptons in $\left(8^{\prime}, 1^{c}\right)$ have the charges $\{2,1,1,1,0,0,0,-1\}$, which are minus the charges of the leptons in $\left(8,1^{c}\right)$. The quarks in $\left(8,3^{c}\right)$ have charges $\{5 / 3,2 / 3,2 / 3,2 / 3,-1 / 3,-1 / 3,-1 / 3,-4 / 3\}$, and the antiquarks in $\left(8^{\prime}, \overline{3}^{c}\right)$ have minus those charges. The bosons are listed in Eq. (2.18) with $n_{3}=1$ and $n_{1}=8$ :

$$
\begin{aligned}
91 & =\left(28,1^{c}\right)+\left(1,1^{c}\right)+\left(1,8^{c}\right)+\left(8_{v}, 3^{c}\right)+\left(8_{v}, \overline{3}^{c}\right) \\
& +\left(1,3^{c}\right)+\left(1, \overline{3}^{c}\right),
\end{aligned}
$$

where $8_{v}$ is the vector representation of $\mathrm{SO}_{8}$. From Eqs . (2.15) and (2.16), we see that the $\left(8_{v}, 3^{c}\right)$ and $\left(8_{v}, \overline{3}^{c}\right)$ bo- 
TABLE VII. Boson-fermion couplings in $\mathrm{SO}_{n}$ model where fermions are assigned to the spinor representation. The local generator used in defining $A$ generates the explicit $\mathrm{U}_{1}$ in Eq. (2.20) or Eq. (2.23). A is the adjoint representation of $\mathrm{SO}_{n-6}$, the $(n-6)(n-7) / 2$.

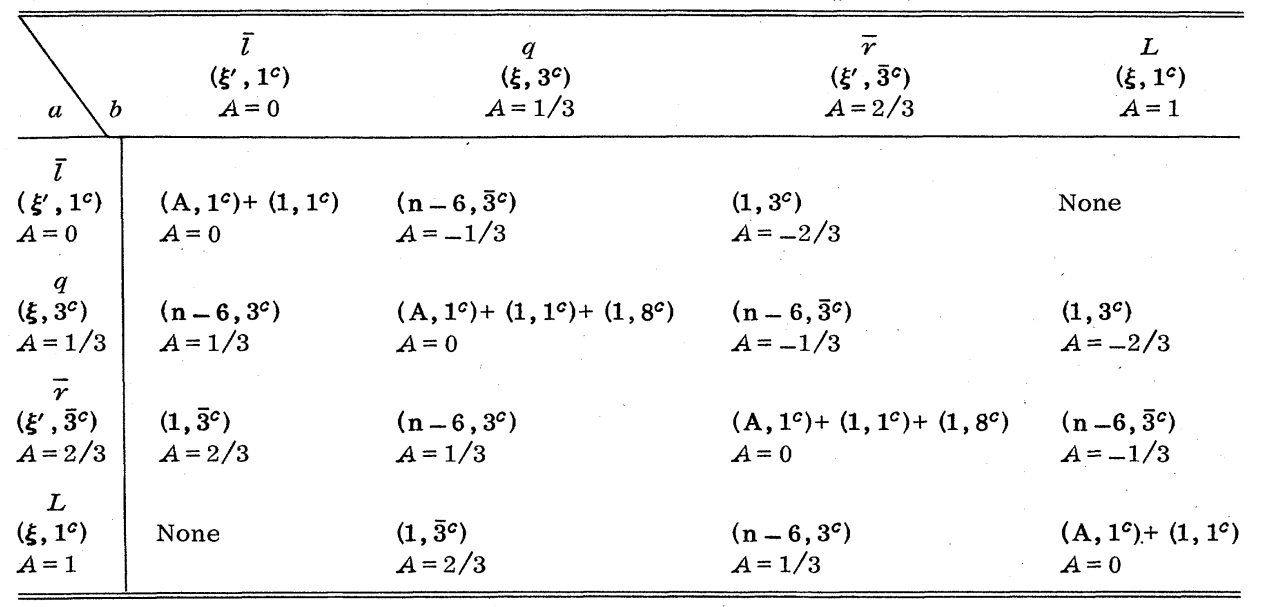

sons provide the transitions, $\bar{l} \rightarrow q, q-r$, and $r \rightarrow L$, where $L$ are leptons assigned to $\left(8,1^{c}\right), q$ to $\left(8,3^{c}\right), \bar{l}$ to $\left(8^{\prime}, 1^{c}\right)$, and $\bar{r}$ to $\left(8^{\prime}, \overline{3}^{c}\right)$. Those bosons act as the $\left(5,3^{c}\right)$ and $\left(5, \overline{3}^{c}\right)$ bosons do in the $\mathrm{SU}_{8}$ example. There is a difference however, because the $\mathrm{SO}_{14}$ example has $\left(1,3^{c}\right)$ and $\left(1, \overline{3}^{c}\right)$ bosons in addition, and those connect $L \hookrightarrow q$ and $\bar{l} \longleftrightarrow \bar{r}$.

The proton is rather naturally unstable in this scheme because both $L$ and $\bar{l}$ lepton multiplets may have neutral members, and, for example, an $\left(8_{v}, 1^{c}\right)$ breaking term would mix the neutral $l$ and $L$ members, and the 3 "light" lepton decay mode would be opened.

In the most economical construction of $A$ so far as weirdness is concerned (see Case 5 of Sec. IV), the broken local generator generates the explicit $U_{1}$. Then the weird $L$ leptons have $A=1$ and the weird $r$ quarks have $A=-2 / 3$. This possibility precludes $L-\bar{\tau}$ mixing, and a $\left(8_{v}, 1^{c}\right)$ breaking term must be absent. The color triplet bosons again carry fractional values of $A(A= \pm 1 / 3, \pm 2 / 3)$, but the decay processes for the weird particles are similar to those of the $\mathrm{SU}_{8}$ model. The model is summarized in Table VII.

The flavor chiral theory places the left-handed fermions in the 64 and the right-handed fermions in the $\overline{64}$, where both of these multiplets have fermion number +1 . Thus all the left-handed spin $1 / 2$ states are classified by $64+64$, and all the right-handed spin $1 / 2$ states are in $\overline{64}+\overline{64}$. (The $U_{1}$ symmetry connecting 64 and $\overline{64}$ is generated by a pseudoscalar charge, and cannot be used to construct $A$ ). If $q_{L}$ are assigned to the $\left(8,3^{c}\right)$, then $q_{R}$ must be assigned to the $\left(8^{\prime}, 3^{c}\right)$ in the $\overline{64}$ in order for $q_{L}$ and $q_{R}$ to have the same charge, color, and baryon number. At this point, the analysis of proton stability becomes identical to the vectorlike case, where $q_{L}$ and $q_{R}$ both transform as $\left(8,3^{c}\right)$. However, one expects the analysis of the full symmetry breakdown of vectorlike and flavor chiral theories to differ in many details.

As our final example, we look at an $E_{7}$ model. The exceptional group models without fermion number predict proton decay in second order, even before the symme- tries are violated by the process $(q q q) \rightarrow(\bar{q} q l)$. For a proton-stable $E_{7}$ model, we double the fermion representation. There is no natural local $\mathrm{U}_{1}$. Instead the $\mathrm{U}_{1}$ must be raided from $G^{\mathrm{f} 1}=\mathrm{SU}_{6}$. Of course such a flavor raid may be made in nonexceptional group models, but here it is mandatory. As discussed in Sec. IV, we take the $\mathrm{U}_{1}$ from $\mathrm{SU}_{6} \supset \mathrm{SU}_{5} \times \mathrm{U}_{1}$, and obtain a rich spectrum of weird particles, Eq. (4.20). This scheme has $\operatorname{six} A$ $=1 / 3$ quarks, of which one $Q=-1 / 3$ quark is separated from the other three with that charge. (See Table VIII.) The phenomenology is expected to be quite similar to that of the proton unstable theory (Gürsey and Sikivie, 1976, 1977; Ramond, 1976, 1977) based on a single 56 of fermions. We note the similarities of this model and the $\mathrm{SU}_{8}$ model where the fermions are assigned to the 28 , and the embedding is $\mathrm{SU}_{8} \supset \mathrm{SU}_{5} \times \mathrm{U}_{1} \times \mathrm{SU}_{3}^{c}$. The 56 of $E_{7}$ branches to $28+\overline{28}$ of $\mathrm{SU}_{8}$. The results are summarized in Table VIII.

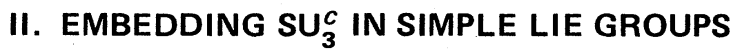

The object in this section is to solve the mathematical problem that arose in the introduction: given a simple Lie group $G$ larger than $\mathrm{SU}_{3}$, find all embeddings of $\mathrm{SU}_{3}$ (identified physically as the color group $\mathrm{SU}_{3}^{c}$ of the strong interactions) for which at least one representation of $G$ contains at most color singlets, triplets, and antitriplets. Then, for each embedding, find all other representations (if there are any) that satisfy the same color restriction. In this article we assign the fermions to such representations, but we recognize that the embeddings may be useful for theories in which the fermions are assigned to other representations.

For any embedding of $\mathrm{SU}_{3}^{c}$ in $G$, the color content of the generators of $G$ is specified, and we may therefore identify the flavor subgroup $G^{f 1}$, which is generated by all the color singlet generators of $G$. The subgroup decomposition is

$$
G \supset G^{\mathrm{f1}} \times \mathrm{SU}_{3}^{c} \text {. }
$$

The generators of $G^{\mathrm{fl}}$ include, of course, the weak 
TABLE VIII. Boson-fermion couplings in the $E_{7}$ model, fermions in the 56. The $\mathrm{SU}_{6} \times \mathrm{SU}_{3}^{c}$ decompositions of the fermions and bosons are contained in the boxes. We assume $X$ used in stabilizing the proton generates the $\mathrm{U}_{1}$ in $\mathrm{SU}_{6} \supset \mathrm{SU}_{5} \times \mathrm{U}_{1}$. Thus the subdivision of eigenstates of $A$ corresponds to the $\mathrm{SU}_{5} \times \mathrm{SU}_{3}^{c}$ decomposition of the 56 and 133 .

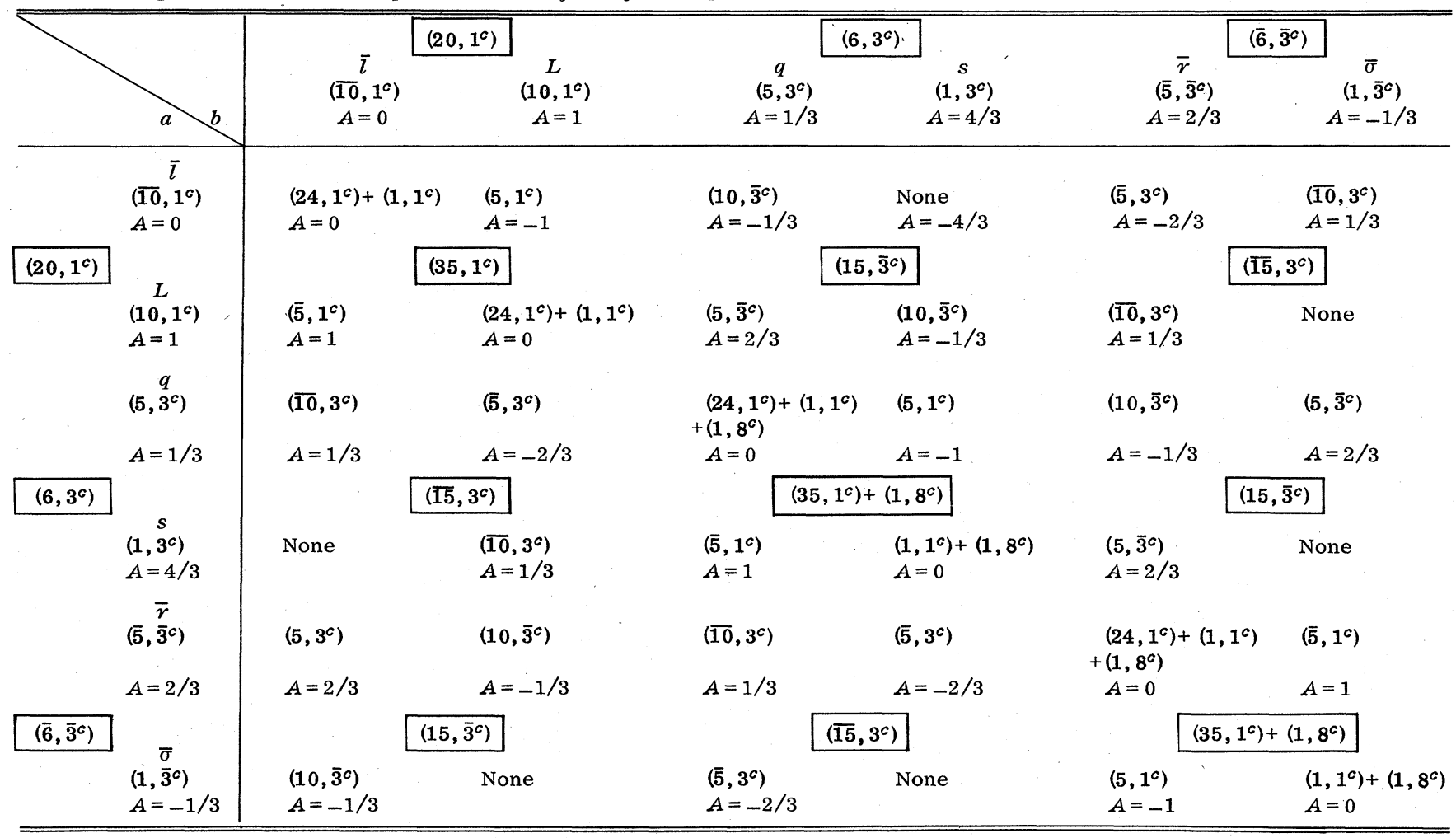

charges and the exactly conserved electric charge $Q$. We are also assuming that the color charges, the generators of $\mathrm{SU}_{3}^{c}$, are exactly conserved. (Occasionally, we shall toy with the idea of enlarging the exactly conserved Yang-Mills group of the strong interactions to the direct product of $\mathrm{SU}_{3}$ and another group, the whole being a subgroup of $G$.)

The restriction on the color content of the fermion representation permits a very simple embedding procedure. We prove in the Appendix the crucial theorem: if any representation $f$ of $G$ decomposed according to Eq. (2.1) contains at most $1^{c}, 3^{c}$, and $\overline{3}^{c}$, then the fundamental representation also contains at most $1^{c}, 3^{c}$, and $\overline{3}^{c}$. (The fundamental representations of the simple Lie groups are: $\mathrm{n}$ of $\mathrm{SU}_{n} ; \mathrm{n}$ of $\mathrm{SO}_{n} ; 2 \mathrm{n}$ of $\mathrm{Sp}_{2 n} ; 7$ of $\mathrm{G}_{2} ; 26$ of $F_{4} ; 27$ of $E_{6} ; 56$ of $E_{7} ;$ and 248 of $\left.E_{8}.\right)$ Thus we may study the embedding in terms of the fundamental representation $\mathrm{n}$, which is not necessarily the fermion representation, with a $G^{\mathrm{f}} \times \mathrm{SU}_{3}^{c}$ decomposition of the form

$$
\mathrm{n}=\left(\mathrm{n}_{1}, 1^{c}\right)+\left(\mathrm{n}_{3}, 3^{c}\right)+\left(\mathrm{n}_{5}, \overline{3}^{c}\right),
$$

where $n_{1}, n_{3}$ and $n_{3}$ are representations of $G^{\mathrm{f} 1}$.

The generators of $G$ belong to the adjoint representation $\operatorname{Adj}(G)$, and its color content is easily obtained from that of the fundamental representation. With the $G^{\text {I1 }}$ $\times \mathrm{SU}_{3}^{c}$ embedding, $\operatorname{Adj}(G)$ has the form

$$
\operatorname{Adj}(G)=\left(\operatorname{Adj}\left(G^{\mathrm{f1}}\right), 1^{c}\right)+\left(1,8^{c}\right)+\text { cross terms },
$$

where the cross terms correspond to the generators of $G$ that mix flavor and color, except in one case for $\mathrm{SU}_{n}$. With $n_{3}$ and $n_{3}$ both different from zero, $\operatorname{Adj}(G)$ includes two $\left(1,8^{c}\right)$ 's, which generate an $\mathrm{SU}_{3} \times \mathrm{SU}_{3}$ subgroup of $\mathrm{SU}_{n}$. The sum of the corresponding generators generates $\mathrm{SU}_{3}^{c}$. There is then a temptation to enlarge the color group to $\mathrm{SU}_{3} \times \mathrm{SU}_{3}$, although doing so is optional. This situation is discussed under Case 3 below. We identify $G^{\mathrm{fl}}$ by explicit examination. It is then straightforward to determine the other representations of $G$ that satisfy our color restrictions.

The whole procedure can be generalized by considering embeddings $G \supset G^{\mathrm{f} 1} \times \mathrm{SU}_{3}^{c}$ with progressively more and more relaxed color restrictions on the fundamental representations and on the others to be used for the fermions.

We carry out this procedure for all simple groups in this section. The results are summarized in Table I, where the group, embeddings, and representations are tabulated. The derivation of these results occupies the remainder of this section. We have also included a short review of each of the Lie algebras. For a more complete review, see, for example, Dynkin (1957), Wybourne (1974), or Gilmore (1974); the tables of Patera and Sankoff (1973) contain useful group theoretic results.

\section{A. Unitary groups}

The special unitary group $\mathrm{SU}_{n}$ is generated by an algebra (called $A_{n-1}$ in the mathematics literature) of rank $n-1$ and order $n^{2}-1$. The geometrical interpretation of $\mathrm{SU}_{n}$ is that it leaves invariant the scalar products of vectors in an $n$-dimensional complex vector space. Its representations are in general complex. The $n-1$ re- 
presentations obtained by taking totally antisymmetric $k$-fold products $\left(\mathrm{n}^{k}\right)_{A}$ are irreducible and of dimension $\left(\begin{array}{l}n \\ k\end{array}\right)$, a binomial coefficient. The conjugate representation of $\left(\mathrm{n}^{k}\right)_{A}$ is equivalent to $\left(\mathrm{n}^{n-k}\right)_{A}$. All other representations are obtained from partially symmetrized products of $n$ with itself. The adjoint representation $n^{2}-1$ is contained in the product $n \times \overline{\mathbf{n}}=\left(\mathrm{n}^{2}-1\right)+1$.

\section{Case 1:}

Because $n$ is complex, the simplest form of Eq. (2.2) is

$$
\mathrm{n}=\left(\mathrm{n}_{1}, 1,1^{c}\right)+\left(1, \mathrm{n}_{3}, 3^{c}\right),
$$

where $n=n_{1}+3 n_{3}$, and $n_{1}$ and $n_{3}$ are integers greater than 1. The notation in Eq. (2.4) reflects the fact that this will be a Class I embedding,

$$
G^{\mathrm{f} 1}=G_{l} \times G_{q} \times \mathrm{U}_{1},
$$

as defined in the Introduction. Since this is a special example of Eq. (2.2) with $n_{\overline{3}}=0$, it will not yield the only embedding. However, as we shall see, the general case has new features that should be discussed separately. The adjoint representation of $\mathrm{SU}_{n}$ provides the list of the generators needed to identify the embedding

$$
\begin{aligned}
\mathrm{n}^{2}-1= & \left(\mathrm{n}_{1}^{2}-1,1,1^{c}\right)+\left(1, \mathrm{n}_{3}^{2}-1,1^{c}\right)+\left(1,1,1^{c}\right)+\left(1,1,8^{c}\right) \\
& +\left(\mathrm{n}_{1}, \overline{\mathrm{n}_{3}}, \overline{3^{c}}\right)+\left(\overline{\mathrm{n}}_{1}, \mathrm{n}_{3}, 3^{c}\right)+\left(1, \mathrm{n}_{3}^{2}-1,8^{c}\right) .
\end{aligned}
$$

Note that Eq. (2.6) includes no flavor cross terms that are color singlets, so that $G^{\mathrm{f1}}$ of Eq. (2.1) is indeed given by

$$
G^{\mathrm{f} 1}=\mathrm{SU}_{n 1} \times \mathrm{SU}_{n 3} \times \mathrm{U}_{1} .
$$

The $U_{1}$ distinguishes $1^{c}$ and $3^{c}$ in the fundamental representation.

For $n_{3}>1$, only the $\mathrm{n}$ of $\mathrm{SU}_{n}$ satisfies the restriction that no more than $1^{c}, 3^{c}$, and $\overline{3}^{c}$ occur in the fermion representation; the leptons are assigned to $\left(n_{1}, 1,1^{c}\right)$ and the quarks to $\left(1, n_{3}, 3^{c}\right)$ of Eq. (2.4). The assignment must be vectorlike in order to avoid divergences from triangle anomalies.

\section{Case 2:}

We may ignore the trivial case $n_{1}=1$ in Eq. (2.4) for which all leptons have the same electric charge, but an interesting special case occurs for $n_{3}=1$ and $n_{1}>1$. Then $\mathrm{n}$ becomes

$$
\mathrm{n}=\left(\mathrm{n}_{1}, 1^{c}\right)+\left(1,3^{c}\right),
$$

with $n_{1}=n-3$. The adjoint representation is

$$
\mathrm{n}^{2}-1=\left(\mathrm{n}_{1}^{2}-1,1^{c}\right)+\left(1,1^{c}\right)+\left(1,8^{c}\right)+\left(\bar{n}_{1}, 3^{c}\right)+\left(\mathrm{n}_{1}, \overline{3}^{c}\right),
$$

which implies the Class III embedding,

$$
G^{\mathrm{f1}}=\mathrm{SU}_{n 1} \times \mathrm{U}_{1} \text {. }
$$

Equation (2.8) by itself is not an interesting candidate for the fermions. However, our color restriction is satisfied for the representations of dimension $\left(\begin{array}{c}n \\ k\end{array}\right)$, obtained by antisymmetrizing $\mathrm{n} k$ times,

$$
\begin{aligned}
\left(\mathrm{n}_{1}^{k}\right)_{A}= & {\left[\left(\mathrm{n}_{1}^{k}\right)_{A}, 1^{c}\right]+\left[\left(\mathrm{n}_{1}^{k-1}\right)_{A}, 3^{c}\right]+\left[\left(\mathrm{n}_{1}^{k-2}\right)_{A}, \overline{3}^{c}\right] } \\
& +\left[\left(\mathrm{n}_{1}^{k-3}\right)_{A}, 1^{c}\right] .
\end{aligned}
$$

The last term is omitted for $k=2$. If $n$ is even and $k$ $=n / 2,\left(\mathrm{n}^{k}\right)_{A}$ is self-conjugate. [For example, the 20 of $\mathrm{SU}_{6}$ is $\left(6^{3}\right)_{A}$, which is equivalent to $\left(\overline{6}^{3}\right)_{A}$.] Otherwise, each of these representations is complex and unsafe from triangle anomaly divergences. There are no other representations of $\mathrm{SU}_{n}$ that satisfy our color restriction.

\section{Case 3:}

If both $n_{3}$ and $n_{\overline{3}}$ of Eq. (2.2) are nonzero, the adjoint contains two color octets and the most natural embedding is not really of the form Eq. (2.1) since the two 8's are generators of separate $\mathrm{SU}_{3}$ 's. The embedding should be written initially as

$$
\mathrm{SU}_{n} \supset\left(\mathrm{SU}_{n 1} \times \mathrm{SU}_{n 3} \times \mathrm{SU}_{n \overline{3}} \times \mathrm{U}_{1} \times \mathrm{U}_{1}\right) \times \mathrm{SU}_{3}^{c \prime} \times \mathrm{SU}_{3}^{c^{\prime \prime}},
$$

where the flavor group has the structure of a Class II embedding

$$
G^{\mathrm{f} 1}=G_{l} \times G_{q} \times G_{\bar{r}} \times \mathrm{U}_{1} \times \mathrm{U}_{1} .
$$

Only the fundamental representation satisfies the color restrictions. The $\mathrm{n}$ contains $n_{1}$ leptons, $n_{3} q$ quarks and $n_{\overline{3}} \bar{r}$ antiquarks, with $n=n_{1}+3 n_{3}+3 n_{\overline{3}}$. Since $\mathrm{n}$ of $\mathrm{SU}_{n}$ is unsafe from triangle anomalies, the fermion assignment must be vectorlike; both $\mathrm{SU}_{3}^{c^{\prime}}$ and $\mathrm{SU}_{3}^{c^{\prime \prime}}$ are generated by vector currents. Consequently there is a temptation to enlarge the color group.

So that both $q$ and $r$ quarks be confined, the conventional color generators must be sums of the corresponding $\mathrm{SU}_{3}^{c^{\prime}}$ and $\mathrm{SU}_{3}^{c^{\alpha}}$ generators. The eight $\mathrm{SU}_{3}^{c}$ generators are conserved, but there are two distinct possibilities for the remaining eight $\mathrm{SU}_{3}^{c^{\prime}} \times \mathrm{SU}_{3}^{c^{\prime \prime}}$ generators: either they are all broken, or they are all conserved. If only $\mathrm{SU}_{3}^{c}$ is conserved we obtain the usual strong interaction gauge group: the $q$ and $r$ quarks would be confined by the same set of gluons, and the hadron spectrum would include $q q r$ and $q \bar{r}$ states. If the unbroken strong gauge group were the full $\mathrm{SU}_{3} \times \mathrm{SU}_{3}$, then the $q$ and $r$ quarks would be bound together by different sets of gluons. Consequently $q \bar{r}$ states, which transform as $(3, \overline{3})$ of color, would be confined; similar considerations apply to the $q q r$ states. The $q q q$ and $q \bar{q}$ hadrons would be quite distinct from the $r r r$ and $r \bar{r}$ ones. Bosons of the unified theory transforming as $(3,3)$ and $(\overline{3}, \overline{3})$ of color would couple $q$ to $r$.

\section{B. Orthogonal groups}

The special orthogonal group $\mathrm{SO}_{m}$ is the group of transformations that leaves invariant the scalar products of vectors in an $m$-dimensional real-vector space. The defining or vector representation is denoted by $\mathrm{v}$ or $\mathrm{m}$, and is self-conjugate. The adjoint representation is obtained irreducibly from $(\mathrm{m} \times \mathrm{m})_{A}$, and has dimension $\frac{1}{2} m(m-1)$. The spinor spaces associated with the algebras differ for $m$ even and $m$ odd.

For an odd-dimensional defining space, the algebra (called $B_{n}$ ) of the group $\mathrm{SO}_{2 n+1}$ has rank $n$ and order $n(2 n+1)$. There is a single real spinor representation $\sigma$ of dimension $2^{n}$, and it cannot be obtained from Kronecker products of $\mathrm{v}$ with itself. However, $\sigma$ is determined up to phases by $\mathbf{v}$, as can be seen from the decomposition of the direct product 


$$
\sigma \times \sigma=\sum_{k=0}^{n}\left(v^{k}\right)_{A}
$$

where $\left(v^{k}\right)_{A}$ are all irreducible $\mathrm{SO}_{2 n+1}$ representations. Note that both the vector and adjoint representations appear in $\sigma \times \sigma$. The assignment of fermions to $\sigma$ obeys our color restriction only for $n_{3}=1$, and so $n_{3}=1$ and $n_{3}$ $>1$ will be considered as separate cases.

In an even number of dimensions, the algebra (called $\left.D_{n}\right)$ of the group $\mathrm{SO}_{2 n}$ has rank $n$ and order $n(2 n-1)$. There are two inequivalent spinors, $\sigma$ and $\sigma^{\prime}$, each of dimension $2^{n-1}$. The Kronecker products of $\sigma$ and $\sigma^{\prime}$ are

$$
\begin{aligned}
& \sigma \times \sigma^{\prime}=\left(\mathrm{v}^{n-1}\right)_{A}+\left(\mathrm{v}^{n-3}\right)_{A}+\ldots \\
& \sigma \times \sigma=\tilde{\sigma}+\left(\mathrm{v}^{n-2}\right)_{A}+\left(\mathrm{v}^{n-4}\right)_{A}+\ldots,
\end{aligned}
$$

where $\tilde{\sigma}$ is a representation of dimension $\frac{1}{2}\left(\begin{array}{c}2 n \\ n\end{array}\right)$, and a formula similar to Eq. (2.16) holds for $\sigma^{\prime} \times \sigma^{\prime}$. . For $n$ even, both $\sigma$ and $\sigma^{\prime}$ are self-conjugate, the vector appears in $\sigma \times \sigma^{\prime}$, and the adjoint in $\sigma \times \sigma$ (and $\sigma^{\prime} \times \sigma^{\prime}$ ). For $n$ odd, $\sigma^{\prime}=\bar{\sigma}$ is the conjugate of $\sigma$, which is complex, and $\sigma \times \bar{\sigma}$ contains the adjoint representation.

We should mention the equivalence of the algebras of $\mathrm{SO}_{6}$ and $\mathrm{SU}_{4}$, of $\mathrm{SO}_{5}$ and $\mathrm{Sp}_{4}$, and also of $\mathrm{SU}_{2}, \mathrm{SO}_{3}$, and $S p_{2}$.

\section{Case 4:}

The fundamental representation of $\mathrm{SO}_{n}$ is the vector representation $\mathrm{n}$, where $\mathrm{n}$ may be even or odd. Since $\mathrm{n}$ is self-conjugate, $3^{c}$ and $\overline{3}^{c}$ must appear symmetrically, and Eq. (2.2) must be of the form

$$
\mathrm{n}=\left(\mathrm{n}_{1}, 1,1^{c}\right)+\left(1, \mathrm{n}_{3}, 3^{c}\right)+\left(1, \overline{\mathrm{n}}_{3}, \overline{3}^{c}\right),
$$

where $n=n_{1}+6 n_{3}$ and $\left(\mathrm{n}_{1}, 1\right)$ is a self-conjugate representation of $G^{\mathrm{f} 1}$. Here we consider $n_{1}$ and $n_{3}$ greater than 1 ; as our notation indicates, this is a Class I embedding.

$G^{\mathrm{f} 1}$ is identified from the adjoint representation

$$
\begin{aligned}
(\mathrm{n} \times \mathrm{n})_{A}= & {\left[\frac{1}{2} \mathrm{n}_{1}\left(\mathrm{n}_{1}-1\right), 1,1^{c}\right]+\left(1, \mathrm{n}_{3}^{2}-1,1^{c}\right)+\left(1,1,1^{c}\right) } \\
& +\left(1,1,8^{c}\right)+\left(\mathrm{n}_{1}, \mathrm{n}_{3}, 3^{c}\right)+\left(\mathrm{n}_{1}, \overline{\mathrm{n}}_{3}, \overline{3}^{c}\right) \\
& +\left[1, \frac{1}{2} \mathrm{n}_{3}\left(\mathrm{n}_{3}+1\right), \overline{3}^{c}\right]+\left[1, \overline{\frac{1}{2} \mathrm{n}_{3}\left(\mathrm{n}_{3}+1\right)}, 3^{c}\right] \\
& +\left[1, \frac{1}{2} \mathrm{n}_{3}\left(\mathrm{n}_{3}-1\right), 6^{c}\right]+\left[1, \overline{\frac{1}{2} \mathrm{n}_{3}\left(\mathrm{n}_{3}-1\right)}, \overline{6}^{c}\right],
\end{aligned}
$$

and the flavor group is

$$
G^{11}=\mathrm{SO}_{n 1} \times \mathrm{SU}_{n 3} \times \mathrm{U}_{1} \text {. }
$$

The explicit $U_{1}$ in Eq. (2.19) counts $3^{c}$ 's minus $\overline{3}^{c}$ 's in Eq. (2.17), and has zero eigenvalue for the color singlet part of $\mathrm{n}$. There are no other representations of $\mathrm{SO}_{n}\left(n_{3}>1\right)$ that satisfy our color restriction.

\section{Case 5:}

Although the structure of the spinor representations of $\mathrm{SO}_{m}$ differs for $m$ even and odd, the characterization of their color content is similar enough to treat them together. Recall that we embed $\mathrm{SU}_{3}^{c}$ through the fundamental representation, Eq. (2.17). The requirement that the spinor representation contain $1^{c}, 3^{c}$, and $\overline{3}^{c}$ only implies the same for $m$, as proved in the Appendix. We first show that $n_{3}=1$ in Eq. (2.17), so $G^{11}=\mathrm{SO}_{m-6} \times \mathrm{U}_{1}$; we then give the $\mathrm{SO}_{m-6} \times \mathrm{SU}_{3}^{c}$ decomposition of the spinors.

First consider $\mathrm{SO}_{2 n+1}$. Since the spinor of $\mathrm{SO}_{2 n+1}$ has only $1^{c}, 3^{c}, \overline{3}^{c}$, the color content of $\sigma \times \sigma$ cannot go beyond $1^{c}, 3^{c}, \overline{3}^{c}, 6^{c}, \overline{6}^{c}, 8^{c}$. However, the color content of $\sigma \times \sigma$ for the embedding can be computed directly from Eqs. (2.14) and (2.17). Specifically, if $n_{3}>1,\left(v^{3}\right)_{A}$ contains a $15^{c}$, so $\sigma$ can satisfy the color restriction only if $n_{3}=1$ in Eq. (2.17). Since the factor $\mathrm{SU}_{n 3}$ disappears for $n_{3}=1$, the flavor group is

$$
G^{\mathrm{f1}}=\mathrm{SO}_{2 n-5} \times \mathrm{U}_{1} \text {. }
$$

The decomposition of the $\mathrm{SO}_{2 n+1}$ spinor into $\mathrm{SO}_{2 n+1-2 j}$ $\times \mathrm{SO}_{2 j}$ representations is particularly simple

$$
\sigma=(\xi, \rho)+\left(\xi, \rho^{\prime}\right),
$$

where $\xi$ is the $\mathrm{SO}_{2 n+1-2 j}$ spinor, and $\rho$ and $\rho^{\prime}$ are the two $\mathrm{SO}_{2 j}$ spinors. We set $j=3$, so that $\rho$ and $\rho^{\prime}$ are the 4 and $\overline{4}$ of $\mathrm{SO}_{6} \approx \mathrm{SU}_{4}$. When $\mathrm{SU}_{4}$ is broken down to $\mathrm{U}_{1} \times \mathrm{SU}_{3}^{c}, 4$ decomposes into $1^{c}+3^{c}$ and the decomposition of $\sigma$ is

$$
\sigma=\left(\xi, 1^{c}\right)+\left(\xi, 3^{c}\right)+\left(\xi, 1^{c}\right)+\left(\xi, \overline{3}^{c}\right) .
$$

The proof that the color restriction on $\sigma$ requires $n_{3}=1$ works also for the spinors of $\mathrm{SO}_{2 n}$. For $n$ odd, $\sigma$ is complex and $\sigma^{\prime}=\bar{\sigma}$, so that neither $\sigma \times \bar{\sigma}$ nor $\sigma \times \sigma$ can have color representations of dimension greater than 8 . Equations (2.15) and (2.16) then imply that $n_{3}=1$ in Eq. (2.17). The same argument applies if $n$ is even, although $\sigma$ and $\sigma^{\prime}$ are then self-conjugate spinors and only $\sigma \times \sigma$ needs to satisfy the color restriction. The flavor group is then

$$
G^{\mathrm{i} 1}=\mathrm{SO}_{2 n-6} \times \mathrm{U}_{1},
$$

and we have a Class III embedding.

The $\mathrm{SO}_{2 n-6} \times \mathrm{SO}_{6}$ decompositions of the $\mathrm{SO}_{2 n}$ spinors are

$$
\begin{aligned}
& \sigma=(\xi, 4)+\left(\xi^{\prime}, \overline{4}\right), \\
& \sigma^{\prime}=\left(\xi^{\prime} 4\right)+(\xi, \overline{4}),
\end{aligned}
$$

and the $G^{\mathrm{f} 1} \times \mathrm{SU}_{3}^{c}$ decompositions are

$$
\begin{aligned}
& \sigma=\left(\xi, 1^{c}\right)+\left(\xi, 3^{c}\right)+\left(\xi^{\prime}, 1^{c}\right)+\left(\xi^{\prime}, \overline{3}^{c}\right), \\
& \sigma^{\prime}=\left(\xi^{\prime}, 1^{c}\right)+\left(\xi^{\prime}, 3^{c}\right)+\left(\xi, 1^{c}\right)+\left(\xi, \overline{3}^{c}\right),
\end{aligned}
$$

where $\xi$ and $\xi^{\prime}$ are $\mathrm{SO}_{2 n-6}$ spinors. As before, the $\mathrm{U}_{1}$ of Eq. (2.23) appears in the decomposition $\mathrm{SU}_{4} \supset \mathrm{SU}_{3}^{c} \times \mathrm{U}_{1}$.

There are no other representations of $\mathrm{SO}_{m}$ that satisfy the color restriction, since $\sigma, \sigma^{\prime}$ and $\mathrm{v}$ each contain both $3^{c}$ and $\overline{3}^{c}$.

\section{Symplectic groups}

The symplectic algebra (called $C_{n}$ ) generates the group $s p_{2 n}$ of transformations that leave invariant a skew-symmetric quadratic form in a real $2 n$-dimensional vector space. It is of rank $n$ and of order $n(2 n+1)$. All of its representations are self-conjugate and may be obtained from Kronecker products of the $2 n$-dimensional defining representation with itself. The adjoint is the symmetric product of $2 n$ with itself. The products $\left(2 n^{k}\right)_{s}$ are irreducible, while $\left(2 n^{k}\right)_{A}$ is a sum of two irreducible representations, one of dimension $\left(\begin{array}{c}2 n \\ k\end{array}\right)-\left(\begin{array}{c}n \\ k-2\end{array}\right)$ and the other of dimension $\left(\begin{array}{c}2 n \\ k-2\end{array}\right)$. Since $2 n$ is real, $3^{c}$ and $\overline{3}^{c}$ must appear symmetrically.

\section{Case 6:}

The most general form of $2 \mathrm{n}$ consistent with Eq. (2.2) is 


$$
2 \mathrm{n}=\left(2 \mathrm{n}_{1}, 1,1^{c}\right)+\left(1, \mathrm{n}_{3}, 3^{c}\right)+\left(1, \overline{\mathrm{n}}_{3}, \overline{3}^{c}\right),
$$

where $n=n_{1}+3 n_{3}$. Because both 3 and $\overline{3}^{c}$ appear in $2 \mathrm{n}$, all higher representations have at least $8^{c}$ and are excluded for fermions by our color restriction. The adjoint representation is

$$
\begin{aligned}
\mathrm{n}(2 \mathrm{n}+1)= & {\left[\mathrm{n}_{1}\left(2 \mathrm{n}_{1}+1\right), 1,1^{c}\right]+\left(1, \mathrm{n}_{3}^{2}-1,1^{c}\right)+\left(1,1,1^{c}\right) } \\
& +\left(1,1,8^{c}\right)+\left(2 \mathrm{n}_{1}, \mathrm{n}_{3}, 3^{c}\right)+\left(2 \mathrm{n}_{1}, \overline{\mathrm{n}}_{3}, \overline{3}^{c}\right) \\
& +\left[1, \frac{1}{2} \mathrm{n}_{3}\left(\mathrm{n}_{3}+1\right), 6^{c}\right]+\left[1, \overline{\frac{1}{2} \mathrm{n}_{3}\left(\mathrm{n}_{3}+1\right)}, \overline{6}^{c}\right] \\
& +\left[1, \frac{1}{2} \mathrm{n}_{3}\left(\mathrm{n}_{3}-1\right), \overline{3}^{c}\right]+\left[1, \overline{\frac{1}{2} \mathrm{n}_{3}\left(\mathrm{n}_{3}-1\right)}, 3^{c}\right] \\
& +\left(1, \mathrm{n}_{3}^{2}-1,8^{c}\right),
\end{aligned}
$$

which implies the Class I embedding,

$$
G^{\mathrm{f} 1}=S p_{2 n_{1}} \times \mathrm{SU}_{n_{3}} \times \mathrm{U}_{1} .
$$

For $n_{3}=1$ this embedding is formally of Class III, but physically trivial since the $2 n$ only obeys the color restriction.

\section{Exceptional groups}

Besides the four infinite sequences of simple classical Lie groups, there are five exceptional groups, which do not have the simple geometrical interpretations reviewed above. Instead each is the invariance group of the multiplication table of certain matrices with elements that belong to the nonassociative octonion algebra. It is difficult to build Lie algebras from underlying nonassociative systems, and so their number is limited. These groups have some apparent advantages for particle physics. Quark-lepton universality is assured since the flavor group, which is in Class IV, contains no $U_{1}$ factor to distinguish color triplets from singlets. Also, there is a natural $\mathrm{SU}_{3}^{c}$, which is the subgroup of the automorphism group $G_{2}$ of the underlying octonion algebra that leaves one of its elements invariant. (Of course $\mathrm{SU}_{3}^{c}$ might be a different subgroup of $G$, and we consider that possibility also.)

Two of the exceptional groups fall outside our assumptions. $G_{2}$ has rank 2 , and $\mathrm{SU}_{3}$ alone is a maximal subgroup; thus $G^{11}$ is trivial, lacking even a $U_{1}$ for electromagnetism. $E_{8}$ has rank 8 and 248 generators. It is the only Lie group for which the smallest representation is the adjoint; there are no representations satisfying our color restriction.

We have listed for reference the Kronecker products of the fundamental and adjoint representations of the exceptional groups in Table IX.

\section{Case 7:}

$F_{4}$ has rank 4 and 52 generators. $\mathrm{SU}_{3}^{c}$ is embedded by

$$
F_{4} \supset \mathrm{SU}_{3} \times \mathrm{SU}_{3}^{c},
$$

so that $G^{\mathbf{1}}=\mathrm{SU}_{3}$. Only the smallest nontrivial representation satisfies the color restrictions. Like all other representations of $F_{4}$, it is self-conjugate:

$$
26=\left(8,1^{c}\right)+\left(3,3^{c}\right)+\left(\overline{3}, \overline{3}^{c}\right) .
$$

If the other $\mathrm{SU}_{3}$ were the color group, the re would be no $F_{4}$ representations satisfying our color constraint. (This is proved in the Appendix.) $\mathrm{The}_{3} \mathrm{SU}_{3} \times \mathrm{SU}_{3}^{c}$ decomposition
TABLE IX. Decomposition of products of fundamental and adjoint representations of $G_{2}, F_{4}, E_{6}, E_{7}$, and $E_{8}$.

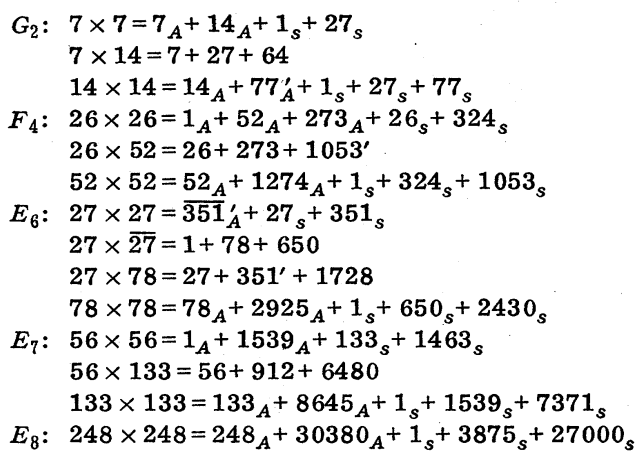

of the adjoint representation is

$$
52=\left(8,1^{c}\right)+\left(1,8^{c}\right)+\left(\overline{6}, 3^{c}\right)+\left(6, \overline{3}^{c}\right) .
$$

\section{Case 8:}

$E_{6}$ has rank 6 and 78 generators. Its fundamental representation is complex, and decomposes as

$$
27=\left(3, \overline{3}, 1^{c}\right)+\left(1,3,3^{c}\right)+\left(\overline{3}, 1, \overline{3}^{c}\right)
$$

under the maximal subgroup decomposition

$$
E_{6} \supset\left(\mathrm{SU}_{3} \times \mathrm{SU}_{3}\right) \times \mathrm{SU}_{3}^{c} \text {. }
$$

Either of the other $\mathrm{SU}_{3}$ 's could be identified as color, and the results of this paper would be unchanged. The adjoint representation is

$$
\begin{aligned}
78= & \left(8,1,1^{c}\right)+\left(1,8,1^{c}\right)+\left(1,1,8^{c}\right) \\
& +\left(3,3, \overline{3}^{c}\right)+\left(\overline{3}, \overline{3}, 3^{c}\right) .
\end{aligned}
$$

The 27 and $\overline{27}$ are the only representations with $1^{c}, 3^{c}$, and $\overline{3}^{c}$ only.

\section{Case 9:}

$E_{7}$ has rank 7 and 133 generators. The color can be embedded by

$$
E_{7} \supset \mathrm{SU}_{6} \times \mathrm{SU}_{3}^{c} \text {. }
$$

Only the 56 satisfies the color restrictions: its $\mathrm{SU}_{6} \times \mathrm{SU}_{3}^{c}$ decomposition is

$$
56=\left(20,1^{c}\right)+\left(6,3^{c}\right)+\left(\overline{6}, \overline{3}^{c}\right) .
$$

The decomposition of the adjoint representation is

$$
133=\left(35,1^{c}\right)+\left(1,8^{c}\right)+\left(\overline{15}, 3^{c}\right)+\left(15, \overline{3}^{c}\right) .
$$

It is also possible that the $\mathrm{SU}_{3}^{c}$ is embedded in the $\mathrm{SU}_{6}$ subgroup of $E_{7}$. This could happen in two ways: (1) If $\mathrm{SU}_{6} \supset \mathrm{SU}_{3}^{c} \times \mathrm{SU}_{3} \times \mathrm{U}_{1}$, then the 56 decomposes to $27+\overline{27}$ $+1+1$ of $E_{6}$. Only the 56 satisfies our color restrictions; (2) If $\mathrm{SU}_{6} \supset \mathrm{SU}_{3}^{c} \times \mathrm{SU}_{2}$, then no $E_{7}$ representation satisfies our color restrictions, which is proven in the Appendix.

\section{THE ELECTRIC CHARGE OPERATOR}

The vector bosons responsible for mediating the electromagnetic (and weak) interactions are coupled to gen- 
erators of the flavor group $G^{f 1}$; the electric charge $Q$ generates a $U_{1}$ subgroup of $G^{\mathrm{f1}}$. The quarks have fractional electric charges in the sequence $(. .5 / 3,2 / 3$, $-1 / 3,-4 / 3, \ldots)$ and the leptons have integral charges $(\ldots, 1,0,-1, \ldots)$. In this section we construct all possible charge operators consistent with these restrictions. The "standard charge assignment," where the quark charges are restricted to $2 / 3$ and $-1 / 3$ and the lepton charges to 0 and -1 , is always possible, although the number of fermions of each charge depends on $G^{f 1}$, but of course other charge assignments are also possible. We proceed by constructing the charge operator for each of the four classes of $G^{\mathrm{f} 1}$ identified in the Introduction. The charge operator in Class I and II theories is only slightly constrained; little discussion is needed. The restrictions for exceptional groups are so tight that possible charge assignments may be listed. Only Class III theories require any effort.

Class I: $G^{\mathrm{f1}}=G_{\imath} \times G_{q} \times \mathrm{U}_{1}$ (Cases 1,4 , and 6 of Table I and Sec. II).

Since the quarks and leptons are transformed by different factors of $G^{\mathfrak{f 1}}$, the observed universality of their weak and electromagnetic charges must result from the mechanism that breaks down $G$ to $\mathrm{SU}_{3}^{c} \times \mathrm{U}_{1}$. This is reflected in the freedom in defining $Q$, which may be written in the general form

$$
Q=\alpha I_{l}+\beta I_{\alpha}+\gamma I_{0},
$$

where $I_{l}$ generates a $\mathrm{U}_{1}$ in $G_{l}, I_{q}$ generates a $\mathrm{U}_{1}$ in $G_{q}$, and $I_{0}$ generates the explicit $U_{1}$. We shall simply enumerate all the constraints on the eigenvalues of $Q$.

\section{Case 1:}

There is only one constraint on the charges of the fermions in the $\mathrm{n}$ of $\mathrm{SU}_{n}$ : the sum of all quark charges (of all colors) is minus the sum of lepton charges, which is proportional to $\gamma$ in Eq. (3.1). For the standard charge assignment, this constraint becomes the number of $Q$ $=-1$ leptons, which equals the number of $Q=2 / 3$ quarks minus the number of $Q=-1 / 3$ quarks; in this special case, the number of quark flavors is equal to the number. of lepton flavors.

\section{Cases 4 and 6:}

The vector representations $\mathrm{n}$ of $\mathrm{SO}_{n}$ and $2 \mathrm{n}$ of $S p_{2 n}$ are self-conjugate. If a single $\mathrm{n}$ of $\mathrm{SO}_{n}$, say, contains both fermions and their antiparticles, then there is complete freedom in defining the fermion charges. It is also possible that $\mathrm{n}$ [Eqs. (2.17) or (2.26)] contains quarks and antiquarks ( $q$ and $\bar{r}$ ) such that $q$ and $r$ a re not equivalent. CPT invariance then requires another fermion representation $\mathrm{n}$ containing the $\bar{q}$ and $r . I_{0}$ counts the number of $q$ 's minus the number of $\bar{r}$ 's. The constraints on the charges are: the sum of the charges in $\left(n, 1,1^{c}\right)$ of $n$ is zero, and the sum of $q$ charges is proportional to $\gamma$ and is equal to the sum of $r$ charges.

Class II: $G^{\mathrm{f} 1}=G_{l} \times G_{\alpha} \times G_{\bar{r}} \times U_{1} \times U_{1}$ (Case 3).

The only constraint on the electric charges is that the sum over all fermion charges in $\mathbf{n}$ must be zero, as in Case 1 of Class I.
Class III: $G^{\mathrm{f1}}=G_{l+q} \times U_{1}$ (Cases 2 and 5).

The generators of $G_{l+\alpha}$ act both on quarks and leptons, and the $U_{1}$ distinguishes $1^{c}$ from $3^{c}$. Thus the quark charges determine the lepton charges, although quarks and leptons sometimes belong to different representations of $G_{q+l}$ so the patterns need not be identical. The most general form of $Q$ is

$$
Q=\alpha I+\beta I_{0},
$$

where $\alpha I$ generates a $U_{1}$ in $G_{q+l}$, and $I_{0}$ generates the explicit $U_{1}$ factor. The value of $\beta$ is determined largely by the fractional nature of the quark charges, and the integer spacing of the charge values is controlled by $\alpha$.

\section{Case 2:}

The charge operator for Class III $\mathrm{SU}_{n}$ theories is a linear combination of an $\mathrm{SU}_{n_{1}}$ generator $\left(n_{1}=n-3\right)$ and the generator $I_{0}$ of the explicit $\mathrm{U}_{1}$. When acting on $\mathrm{n}$ in $\mathrm{Eq}$. (2.8), $I_{0}$ has eigenvalue $1 / n_{1}$ for $\left(n_{1}, 1^{c}\right)$ and $-1 / 3$ for $\left(1,3^{c}\right)$. The eigenvalues of the $\mathrm{SU}_{n_{1}}$ generator may be parametrized by a set of $n_{1}$ integers, $\left\{\Re_{\alpha}^{1}\right\} \equiv\left\{m_{\alpha}\right\}$ $=\left\{m_{1}, m_{2}, \ldots, m_{n_{1}}\right\}$, because of the integral spacing of the electric charges. We define $M=\sum_{\alpha=1}^{n_{1}} m_{\alpha}$, and the eigenvalues of this generator are then $m_{\alpha}-\left(1 / n_{1}\right) M$ when acting on $\left(\mathrm{n}_{1}, 1^{c}\right)$ and are 0 for $\left(1,3^{c}\right)$. There is no loss of generality if we require $0 \leqslant M<n_{1}$.

The fermions are assigned to $\left(n^{k}\right)_{A}$, Eq. (2.11). Thus it is helpful to define $\left\{\mathfrak{T}_{\alpha}^{k}\right\}$ as the set of $\left(\begin{array}{c}n_{1} \\ k\end{array}\right)$ integers obtained by summing $k$ different $m$ 's in all different ways. If we denote the charges in $\left[\left(\mathrm{n}_{1}^{k}\right)_{A}, 1^{c}\right]$ by $Q_{\alpha}\left[\left(\mathrm{n}_{1}^{k}\right)_{A}, 1^{c}\right]$, etc., then the electric charges of the fermions may be parametrized as

$$
\begin{aligned}
& Q_{\alpha}\left[\left(\mathrm{n}_{1}^{k}\right)_{A}, 1^{c}\right]=\mathfrak{T}_{\alpha}^{k}+r, \quad \alpha=1, \ldots,\left(\begin{array}{c}
n_{1} \\
k
\end{array}\right), \\
& Q_{\alpha}\left[\left(\mathrm{n}_{1}^{k-1}\right)_{A}, 3^{c}\right]=\Re_{\alpha}^{k-1}+s-1 / 3, \quad \alpha=1, \ldots,\left(\begin{array}{c}
n_{1} \\
k-1
\end{array}\right), \\
& Q_{\alpha}\left[\left(\mathrm{n}_{1}^{k-2}\right)_{A}, \overline{3}^{c}\right]=\mathfrak{T}_{\alpha}^{k-2}+2 s-r-2 / 3, \quad \alpha=1, \ldots,\left(\begin{array}{c}
n_{1} \\
k-2
\end{array}\right), \\
& Q_{\alpha}\left[\left(\mathrm{n}_{1}^{k-3}\right)_{A}, 1^{c}\right]=\mathfrak{T}_{\alpha}^{k-3}+3 s-2 r-1, \quad \alpha=1, \ldots,\left(\begin{array}{c}
n_{1} \\
k-3
\end{array}\right),
\end{aligned}
$$

where $r$ and $s$ must be integers, and must also satisfy the constraint

$$
(3 k-n) r=(M+3 s-1) k \text {. }
$$

[The parameter $\beta$ of Eq. (3.2) is determined in terms of $r$ and also in terms of $s$, thus giving rise to Eq. (3.4).]

Let us examine some examples of Eqs. (3.3) and (3.4). If we require that $\left\{\mathfrak{T}_{\alpha}^{k}\right\}$ contain two values only, then there are two possibilities for $\left\{m_{\alpha}\right\}$ :

(a) $\left\{m_{\alpha}\right\}=\{1,0, \ldots, 0\}$. If $\left\{Q_{\alpha}\left[\left(\mathbf{n}^{k}\right)_{A}, 1^{c}\right]\right\}$ contains charges $Q=0$ and $Q=1$ (antileptons), then $r=s=0$, and the standard charge assignment is recovered. The particles are as follows:

$\left[\left(\mathrm{n}_{1}^{k}\right)_{A}, 1^{c}\right]$ antileptons, $\left(\begin{array}{c}n-4 \\ k-1\end{array}\right)$ with $Q=1$ and $\left(\begin{array}{c}n-4 \\ k\end{array}\right)$ with $Q=0$; $\left[\left(\mathbf{n}_{1}^{k-1}\right)_{A}, 3^{c}\right]$ quarks,

$\left(\begin{array}{c}n-4 \\ k-2\end{array}\right)$ with $Q=2 / 3$ and $\left(\begin{array}{c}n-4 \\ k-1\end{array}\right)$ with $Q=-1 / 3$;

$\left[\left(\mathrm{n}_{1}^{k-2}\right)_{A}, \overline{3}^{c}\right]$ antiquarks,

$$
\left(\begin{array}{c}
n-4 \\
k-3
\end{array}\right) \text { with } Q=1 / 3 \text { and }\left(\begin{array}{c}
n-4 \\
k-2
\end{array}\right) \text { with } Q=-2 / 3 \text {; }
$$

$\left[\left(\mathrm{n}_{1}^{k-3}\right)_{A}, 1^{c}\right]$ leptons, $\left(\begin{array}{c}n-4 \\ k-4\end{array}\right)$ with $Q=0$ and $\left(\begin{array}{c}n-4 \\ k-3\end{array}\right)$ with $Q=-1$.

(b) The standard charge assignment may also be ob- 
tained in a different way from $\left\{m_{\alpha}\right\}=\{0,1, \ldots, 1\}$ but only for $n$ even and $k=n / 2$.

Larger spreads of electric charge are always possible, although Eq. (3.4) helps to limit the number of cases. As an interesting example, consider $\left\{m_{\alpha}\right\}=\{1,1,0,0, \ldots, 0\}$, with the requirement that the charges $Q_{\alpha}\left[\left(n_{1}^{k}\right)_{A}, 1^{c}\right]$ have the values $1,0,-1$. Then $r=-1$ and $s=1 / 3[(n / k)-4]$, which implies that $n=4 k, 7 k, 10 k$, etc. For the case $n=8$ and $k=2$, there is a quark singlet with $Q=-1 / 3$. This charge assignment for the 28 of $\mathrm{SU}_{8}$ is related to the standard one for the 56 of $E_{7}$ (see Case 9 below); the $\mathrm{SU}_{8}$ decomposition of the 56 of $E_{7}$ is $28+\overline{28}$.

\section{Case 5:}

The charge operator of a Class III $\mathrm{SO}_{n}$ theory is a linear combination of an $\mathrm{SO}_{n-6}$ generator and the generator of the explicit $U_{1}$ in Eq. (2.20) or (2.23). (We treat even and odd $n$ together.) The eigenvalues of the $\mathrm{SO}_{n-6}$ generator depend on $m \equiv[(n-6) / 2]$ parameters, which may be chosen to be integers because of the spacing of the electric charges. The eigenvalues of this generator acting on the $\left(\mathrm{n}-6,1^{c}\right)$ are $\pm n_{1}, \pm n_{2}, \ldots, \pm n_{m},(0)$, where $n_{1}, \ldots, n_{m}$ are non-negative integers and the extra zero eigenvalue is present if $n$ is odd. The eigenvalues of this generator when acting on the spinor representation(s) a re then given by the set $\mathfrak{T}$, where

$$
\mathfrak{T}=\left\{\frac{1}{2}\left( \pm n_{1} \pm n_{2} \pm \cdots \pm n_{m}\right)\right\}
$$

Equation (3.5) is the solution of Eq. (2.14) or Eqs. (2.15) and (2.16). It is most easily derived from the Clifford algebra structure of the $\mathrm{SO}_{n-6}$ spinors, but it may also be constructed using the $\mathrm{SU}_{m} \times \mathrm{U}_{1}$ decomposition of the $\mathrm{SO}_{n-6}$ spinors. For $n$ odd there is only one spinor of dimension $2^{m}$, and the eigenvalues are just the set $\mathfrak{T}$. When $n$ is even there are two $\mathrm{SO}_{n-6}$ spinors, each of dimension $2^{m-1}$ : one has eigenvalues in the subset of $\mathfrak{T}$ with an even number of minus signs, which we call $\mathfrak{M T}_{+}$; and the other has eigenvalues in the subset of $\mathfrak{N}$ with an odd number of minus signs, $\mathfrak{T}$. We also define

$$
N=\frac{1}{2} \sum_{i=1}^{m} n_{i}
$$

All elements of $\mathfrak{T}$ are integer (or half integer) if $N$ is. The sum of the elements of $\mathfrak{T}_{ \pm}$is zero, since $\alpha I$ is traceless in any representation. If $m$ and $n$ are even, then the set $\mathfrak{T}_{ \pm}$is identical to the set $-\mathfrak{T}_{ \pm}$, and for $n$ even and $m$ odd, $\mathfrak{T}_{-}$is identical to $-\mathfrak{M T}_{+}$.

The explicit $\mathrm{U}_{1}$ is obtained from the decomposition $\mathrm{SO}_{6}$ $\approx \mathrm{SU}_{4} \supset \mathrm{U}_{1} \times \mathrm{SU}_{3}^{c}$. Finding the eigenvalues of the generator of the $\mathrm{U}_{1}$ is a simple application of the $\mathrm{SO}_{n-6}$ results that we just obtained. The 6 of $\mathrm{SO}_{6}$ breaks into $3^{c}+\overline{3}^{c}$ of $\mathrm{SU}_{3}^{c}$, and the eigenvalues of the generator of the $U_{1}$ are $\{1,1,1,-1,-1,-1\}$. The eigenvalues of the 4 of $\mathrm{SO}_{6}$, computed from Eq. (3.5), a re then $\frac{1}{2}\{3,-1,-1,-1\}$. We adjust $\beta$ in Eq. (3.2) so that quarks and leptons occur in the appropriate charge sequences. In the case that $N$ in Eq. (3.6) is integral, the sets of electric charges for the fermions in the $\mathrm{SO}_{n}$ spinor representations, Eqs. (2.22) or (2.24) and (2.25), are

$$
\begin{aligned}
& Q_{\alpha}\left(\xi, 1^{c}\right)=\mathfrak{N}_{+\alpha}+3 k+1, \\
& Q_{\alpha}\left(\xi, 3^{c}\right)=\mathfrak{N}_{+\alpha}-k-1 / 3, \\
& Q_{\alpha}\left(\xi^{\prime}, 1^{c}\right)=\mathfrak{M}_{-\alpha}-3 k-1, \\
& Q_{\alpha}\left(\xi^{\prime}, \overline{3}^{c}\right)=\mathfrak{M}_{-\alpha}+k+1 / 3,
\end{aligned}
$$

where the $3 k+1$ is the average charge of the $\left(\xi, 1^{c}\right)$ leptons, etc., and $k$ is an integer. For $n$ odd, $\mathfrak{M}_{+}=\mathfrak{M} \ldots_{-}=\mathfrak{M}$ in Eq. (3.7). The half-integer $N$ case is obtained by replacing $k$ by $k-1 / 2$ in Eq. (3.7) so that

$$
\begin{aligned}
& Q_{\alpha}\left(\xi, 1^{c}\right)=\mathfrak{M}+\alpha+3 k-1 / 2, \\
& Q_{\alpha}\left(\xi, 3^{c}\right)=\mathfrak{M}_{+\alpha}-k+1 / 6, \\
& Q_{\alpha}\left(\xi^{\prime}, 1^{c}\right)=\mathfrak{M}_{-\alpha}-3 k+1 / 2, \\
& Q_{\alpha}\left(\xi^{\prime}, \overline{3}^{c}\right)=\mathfrak{M}_{-\alpha}+k-1 / 6 .
\end{aligned}
$$

In both cases, the average lepton charge in $\left(\xi, 1^{c}\right)$ [or $\left(\xi^{\prime}, 1^{c}\right)$ ] is minus three times the average quark charge in $\left(\xi, 3^{c}\right)$ [or $\left(\xi^{\prime}, 3^{c}\right)$, and the spread in the charges is $2 N$.

The standard charge assignment is recovered for $k=0$ in Eq. (3.8), and $\left\{n_{\alpha}\right\}=\left\{n_{1}, \ldots n_{m}\right\}=\{1,0, \ldots, 0\}$ Then half the leptons have $Q=0$ and the other half have $Q=-1$; half the quarks have $Q=2 / 3$ and the other half have $Q$ $=-1 / 3$. In all these four cases of charge and color, there is the same even number of particles. This corresponds to $\alpha I$ being the generator of the $\mathrm{U}_{1}$ in the embedding $\mathrm{SO}_{n-6} \supset \mathrm{SO}_{n-8} \times \mathrm{SO}_{2}=\mathrm{SO}_{n-8} \times \mathrm{U}_{1}$. There are no models with the average lepton charge of $-1 / 2$ and a spread of 2 units of charge. There are three cases if a spread of three units of charge is desired with average lepton charge $-1 / 2$. These correspond to: (a) $\left\{n_{\alpha}\right\}=$ $\{3,0, \ldots, 0\}$, which has charged currents with $\Delta Q=3$ only; (b) $\left\{n_{\alpha}\right\}=\{2,2,0, \ldots, 0\}$; and (c) $\left\{n_{\alpha}\right\}=$

$\{1,1,1,0, \ldots, 0\}$. As a final example, if the average lepton charge is 1 , then the smallest charge spread is 2 , and the only interesting case is $\left\{n_{\alpha}\right\}=\{1,1,0, \ldots, 0\}$. Then leptons have charges 0,1 , and 2; and quarks have charges $2 / 3,-1 / 3$, and $-4 / 3$, as can be seen from Eq。 (3.7).

Class IV: $G^{\mathrm{f1}}=G_{l+\alpha}($ Cases 7,8 , and 9)

In the exceptional groups, $G^{\mathrm{f1}}$ acts both on quarks and leptons as a simple flavor group. There are no $U_{1}$ factors that distinguish color. The sum of the quark charges vanishes. Quark-lepton universality is imposed at the level of the gauge group, and the quarks and leptons transform as different representations of $G^{\mathrm{p} 1}$, which are related in such a way that $1 / 3$ integral charge assignments for quarks imply integral charge assignments for leptons.

\section{Case 7:}

The flavor group for $F_{4}$ is $\mathrm{SU}_{3}$, and the fermions are assigned to the 26, Eq. (2.30). The eigenvalues of the electric charge operator when acting on the 3 can be parametrized by $\left(n_{1}-1 / 3, n_{2}-1 / 3,-n_{1}-n_{2}+2 / 3\right)$, where $n_{1}$ and $n_{2}$ are integers. The leptons in $\left(8,1^{c}\right)$ have charges $\left\{ \pm\left(n_{1}-n_{2}\right), \pm\left(2 n_{1}+n_{2}-1\right), \pm\left(2 n_{2}+n_{1}-1\right), 0,0\right\}$. The standard charge assignment, which in this case has $Q$ $= \pm 1$ leptons, is recovered with $n_{1}=n_{2}=0$. 


\section{Case 8:}

The generator of the $\mathrm{U}_{1} \subset \mathrm{SU}_{3} \times \mathrm{SU}_{3}$, which is $G^{\mathrm{f} 1}$ for $E_{6}$, must possess eigenvalues $\left(n_{1}-1 / 3, n_{2}-1 / 3,-n_{1}-n_{2}\right.$ $+2 / 3)$ in the $(1,3)$ representation, and $\left(m_{1}-1 / 3, m_{2}\right.$ $\left.-1 / 3,-m_{1}-m_{2}+2 / 3\right)$ in the $(3,1)$ representation. Then the eigenvalues of $Q$ for the $\left(3, \overline{3}, 1^{c}\right)$ of Eq. (2.32) a re $\left\{m_{1}-n_{1}, m_{1}-n_{2}, m_{2}-n_{1}, m_{2}-n_{2}, m_{1}+n_{1}+n_{2}-1,-m_{1}-m_{2}\right.$ $\left.-n_{1}+1, m_{2}+n_{1}+n_{2}-1,-m_{1}-m_{2}-n_{2}+1, n_{1}+n_{2}-m_{1}-m_{2}^{2}\right\}$. The standard charge assignment, which is the only one that looks interesting, is recovered with $n_{1}=n_{2}=m_{1}=m_{2}$ $=0$.

\section{Case 9:}

The generator of an arbitrary $\mathrm{U}_{1} \subset \mathrm{SU}_{6}$ is specified by five parameters. The quarks are in a 6 of $\mathrm{SU}_{6}$, and the charge eigenvalues are $n_{i}-1 / 3, i=1, \ldots, 6$ with $N$ $=\sum_{i=1}^{6} n_{i}=2$. The charges in the $\left(20,1^{c}\right)$ are then the set $\mathfrak{N}_{3}-1$, where $\mathfrak{N}_{3}$ is the set of all different threefold sums of $n_{i}$. Only if $\left\{n_{\alpha}\right\}=\{1,1,0,0,0,0\}$ do we recover the standard charge assignment. A charge $-4 / 3$ quark occurs in the model with $\left\{n_{\alpha}\right\}=\{1,1,1,0,0,-1\}$.

\section{BARYON NUMBER CONSERVATION}

In unified theories, where both quarks $\left(3^{c}\right)$ and leptons $\left(1^{c}\right)$ are assigned to the same irreducible representation of $G$, there exist vector bosons (leptoquarks) that transform leptons into quarks. This opens the way for proton decay unless there exists a conserved quantum number $A$ for which the proton is the lowest mass state with $A$ $=1$. As emphasized in the Introduction, exact proton stabilization is not required, since there are many ways to increase the proton lifetime beyond the experimental limit. Still, a stable proton does not contradict the data, and it is of interest to examine its implications for the unified theories discussed here.

Empirically, conservation of $A$ does not have a longrange force associated with it. Thus, even though $A$ is an additive quantum number like $Q$, it cannot be a generator of a local $U_{1}$. It is therefore necessary for the Lagrangian to possess more symmetry than the local gauge group $G$. An additional global $U_{1}$ symmetry alone is not adequate either, since its generator can merely count representations of $G$, and cannot distinguish between quarks and leptons contained in one representation. It can help to put off proton decay to higher order, but it cannot make the proton absolutely stable. However, if this global $U_{1}$ and some local $U_{1}$ are both broken in such a way that a linear combination of the generators is conserved, the vector boson acquires a mass (leaving no physical Goldstone boson) and the unbroken linear combination gives an exact conservation law. The emphasis on $U_{1}$ 's reflects the fact that $A$ is an additive quantum number. The Lagrangian should possess at least a global $\mathrm{U}_{1}$ symmetry in addition to $G$. (A possible exception is the generation of conserved topological quantum numbers, but no examples with a shortrange force in three spatial dimensions have yet been given.) As a matter of notation, let $X$ be the generator of the local $\mathrm{U}_{1}$ and $Z$ be the generator of the global $\mathrm{U}_{1}$. In carrying out this study we have neglected possible breaking of $Z$ caused by tunneling effects in the vacuum (instantons). Even in theories where there is violation, the proton decay due to this mechanism is very slow ( t'Hooft, 1976).

We now discuss ways of obtaining $Z$. If the Lagrangian contains only an adjoint representation of vector bosons and one self-conjugate irreducible representation of fermions, there are no global symmetries. Extra multiplets must be added before the Lagrangian acquires an additional global $U_{1}$ symmetry. There are two possibilities:

(1) The Lagrangian acquires an extra phase invariance if the fermion representation is doubled; this global symmetry is generated by fermion number. The fermions $\left(Z_{f}=+1\right)$ are in $f$ (some of the fermions may be antiquarks or antileptons), and their antiparticles $\left(Z_{f}\right.$ $=-1$ ) are in $\bar{f}$. Of course doubling $f$ is necessary if $f$ is a complex representation of $\mathrm{SU}_{n}, \mathrm{SO}_{2 n}$, or $E_{6}$. In the first set of solutions for $A$, derived later in this section, we assume that $Z$ is fermion number. The simplest prototype is a dynamically broken theory with spin 1 and spin $1 / 2$ fields only. The bosons carry $Z=0$, and the fermions belong to an irreducible $f$ with $Z=1$, and the antifermions to $\overline{\mathrm{f}}$ with $Z=-1$. Fermion number and $X$ are broken by a Majorana lepton mass term, which has the form $l l+\overline{l l}$. For simplicity we do not consider Lagrangians with additional global symmetry, arising, say, from additional fields in the Lagrangian or from $f$ being reducible. This generalization, which is straightforward, can provide both a conserved baryon number and lepton number, if desired. We then seek an appropriate $X$ so that

$$
A=Z+X
$$

is a suitable atomic mass number. We find that only for Cases 1 and 3 (see Table I) is it possible for all leptons to have $A=0$ and all quarks to have $A=1 / 3$; other cases always predict weird particles. Simple examples are listed in Table III.

(2) The global $U_{1}$ is a symmetry outside the fermion sector so that $Z$ for the fermions is zero. The prototype theory here is one in which all fermions are assigned to a single irreducible self-conjugate $f$, and explicit Higgs fields have nonzero values of $Z$. If $Z$ and $X$ are broken the re with their sum conserved, some of the spin zero fields will acquire nonzero $A$ (the vacuum expectation of these fields must vanish), the zero-mass boson is made heavy, and the atomic mass number in the fermion sector is simply

$$
A=X \text {. }
$$

Again for simplicity we allow just one global $U_{1}$, so the fermions are assigned to self-conjugate representations. (Cases 1, 3, and 8 are absent.) It is possible to formulate $\mathrm{SO}_{n}$ and $S p_{2 n}$ theories of Class I with a stable proton, but no weird fermions; otherwise weird fermions are required.

Whether the symmetry breaking in an actual theory follows the pattern prescribed here will depend on the specific Lagrangian. Some models might not provide a suitable atomic mass number even though the possibility appears in our classification. However, there is of ten a wide choice of $X^{\prime}$ 's that are satisfactory. 
Since $X$ is violated it must be a generator of $G^{\mathrm{f1}}$. In theories based on the classical groups, $G^{\mathrm{fl}}$ contains an explicit $\mathrm{U}_{1}$ subgroup, and usually its generator is a suitable $X$. (The one exception is $\mathbf{f}=\mathbf{n}$ of $\mathrm{SO}_{n}$ with fermion doubling.) It is also possible to extract part of $X$ from the non-Abelian subgroups of $G^{\mathrm{fl}}$; we call this procedure a flavor raid. At present, flavor raids for classical groups appear somewhat academic, but we indicate how they may be systematically pursued. For the exceptional groups, $X$ must result from a flavor raid since there is no explicit $U_{1}$ factor. Just as in Sec. III, we must construct the eigenvalues of a generator of $U_{1} \subset G^{f 1}$, and many of the techniques needed there will again be useful.

\section{A. Doubled $f$, Majorana mass breaking}

\section{Case 1:}

We use this case to set our notation. Let the fermions be in the $\mathbf{n}$ of $\mathrm{SU}_{n}$, so that

$$
f=\left(1,1^{c}\right)+\left(q, 3^{c}\right) \text {. }
$$

Comparing this with Eq. (2.4), we see that $1=\left(\mathrm{n}_{1}, 1\right)$ and $\mathrm{q}=\left(1, \mathrm{n}_{3}\right)$ are representations of $G^{\mathrm{fl}}$. The global $\mathrm{U}_{1}$ is generated by the fermion number operator

$$
Z=N_{l}+N_{\alpha}
$$

where $N_{q}$ means number of $q$ 's minus number of anti$q$ 's. There are $n_{1}+n_{3}-1$ local $\mathrm{U}_{1}$ 's in $\mathrm{SU}_{n_{1}} \times \mathrm{SU}_{n_{3}} \times \mathrm{U}_{1}$, and the only restriction is that $X$ not be the electric charge operator. However, the only choice of $X$ that does not imply weird fermions is the generator of the explicit $\mathrm{U}_{1}$ in $\mathrm{Eq}$ (2.7):

$$
X=N_{l}-\left(n_{1} / 3 n_{3}\right) N_{q}
$$

The Majorana mass term, which breaks both $X$ and $Z$, has the schematic form $l l+\overline{l l}$ so that $A$ cannot contain $N_{l}$. Thus $A$ is proportional to $Z-X$, and after proper normalization

$$
A=\frac{1}{3} N_{Q} \text {, }
$$

which is readily identified with baryon number, since all leptons have $A=0$, and the proton, which is the lowest-mass 3-quark state, has $A=1$. Lepton number is violated because of the Majorana lepton mass, but this can be avoided in more complicated examples. Any other $X$, which would be obtained from a flavor raid, would have to distinguish different types of quarks and (or) leptons, and therefore would necessarily imply weird particles. The elaboration of these cases is straightforward, but not very instructive.

\section{Case 2:}

The $k$-times antisymmetrized $\mathrm{n}$ of $\mathrm{SU}_{n}$ of Eq. (2.11) may be written

$$
\mathbf{f}=\left(\overline{1}, 1^{c}\right)+\left(\mathrm{q}, 3^{c}\right)+\left(\overline{\mathbf{r}}, \overline{3}^{c}\right)+\left(\mathrm{L}, 1^{c}\right) .
$$

In the simplest scheme $X$ generates the explicit $U_{1}$ of Eq. (2.10), and may be normalized to

$$
X=Z-\frac{n}{k}\left(\frac{1}{3} N_{\alpha}-\frac{2}{3} N_{r}+N_{L}\right)
$$

where the fermion number operator $Z$ is

$$
Z=-N_{l}+N_{\alpha}-N_{r}+N_{L}
$$

With the Majorana mass and the known leptons in $\left(1,1^{\circ}\right)$, we obtain

$$
A=\frac{1}{3} N_{Q}-\frac{-}{3} N_{r}+N_{L} \text {. }
$$

Equation (4.10) implies, for example, the existence of heavy $L$ leptons that decay into ordinary baryons, and three-weird-quark ( $3 r$ ) states with $A=-2$ that could decay into an antideuteron plus a light lepton. These weird particles must be sufficiently massive to avoid conflict with experiment.

The most general $A$ assignment is obtained from a flavor raid on $\mathrm{SU}_{n-3}$. The calculation and solution of the spectrum of $A$ is almost identical to the one for the electric charge in Case 2 of Sec. III. Following the notation defined there, we obtain the solution,

$$
\begin{aligned}
& A_{\alpha}\left[\left(\mathrm{n}_{1}^{k}\right)_{A}, 1^{c}\right]=\Re_{\alpha}^{k}+r, \\
& A_{\alpha}\left[\left(\mathrm{n}_{1}^{k-1}\right)_{A}, 3^{c}\right]=\mathfrak{T l}_{\alpha}^{k-1}+s+1 / 3 \text {, } \\
& A_{\alpha}\left[\left(\mathrm{n}_{1}^{k-2}\right)_{A}, \overline{3}^{c}\right]=\mathfrak{M}\left(_{\alpha}^{k-2}+2 s-r+2 / 3\right. \text {, } \\
& A_{\alpha}\left[\left(\mathrm{n}_{1}^{k-3}\right)_{A}, 1^{c}\right]=\mathfrak{N}{ }_{\alpha}^{k-3}+3 s-2 r+1,
\end{aligned}
$$

where $r$ and $s$ are integers satisfying

$$
(3 k-n) r=(M+3 s+1) k-n Z,
$$

and $Z$ is the fermion number, which must be nonzero. Cataloging $A$ assignments is similar to cataloging electric charge assignments, and is easily carried out for specific cases.

\section{Case 3:}

We write the fermion representation of $\mathrm{SU}_{n}$ in Case 3 as

$$
\mathbf{f}=\left(\mathbf{1}, \mathbf{1}^{c}\right)+\left(\mathbf{q}, 3^{c}\right)+\left(\overline{\mathbf{r}}, \overline{\mathbf{3}}^{c}\right),
$$

where the generators of the unbroken $\mathrm{SU}_{3}^{c}$ are the sums of those of the two $\mathrm{SU}_{3}$ 's of Eq. (2.12). Then with $Z$ $=N_{l}+N_{q}-N_{r}$ and $X=-\left(n_{3}-n_{1}\right) N_{l}+\left(2 n_{3}+n_{1}\right) N_{q}+\left(n_{1}+2 n_{3}\right) N_{r}$, we find

$$
A=\frac{1}{3}\left(N_{q}+N_{r}\right) \text {. }
$$

The choice of $X$ is made from linear combinations of the generators of the two explicit $U_{1}^{\prime}$ 's in such a way as to eliminate weird particles. $X$ can be broken by a Majorana mass only if $n_{3} \neq n_{3}$. The situation is similar if the two $\mathrm{SU}_{3}$ 's are both conserved and confining (i.e., the color group is enlarged to $\mathrm{SU}_{3}^{c^{\prime}} \times \mathrm{SU}_{3}^{c^{\prime}}$ ).

\section{Case 4:}

Let us label the vector representation of $\mathrm{SO}_{n}$, Eq. (2.17), as

$$
\mathbf{f}=\left(1,1^{c}\right)+\left(q, 3^{c}\right)+\left(\overline{\mathbf{r}}, \overline{3}^{c}\right),
$$

where $\mathbf{r}$ and $q$ each belong to the $\mathbf{n}_{3}$ representation of $\mathrm{SU}_{n_{3}}$. The explicit $\mathrm{U}_{1}$ in Eq. (2.19) does not count l's, and cannot be broken by a Majorana mass. If the breaking of this $U_{1}$ were to appear elsewhere in the theory, then it would be possible to obtain $A=\frac{1}{3}\left(N_{q}+N_{r}\right)$, but in this set of models, part of $X$ must come from a flavor raid on $\mathrm{SO}_{n}$; for example, decompose $\mathrm{SO}_{n_{1}} \supset \mathrm{SU}_{n^{\prime}} \times \mathrm{U}_{1}$ for $n_{1}=2 n^{\prime}$. Then $\mathrm{n}_{1}$ of $\mathrm{SO}_{n_{1}}$ decomposes into $\mathbf{n}^{\prime}+\overline{\mathbf{n}}^{\prime}$ of $\mathrm{SU}_{n^{\prime}}$ and the $\mathrm{U}_{1}$ generator distinguishes two kinds of 
leptons, which we call $l$ and $L$. There is then a linear combination of this generator and the explicit $U_{1}$ generator such that

$$
A=N_{L}+\frac{1}{3} N_{q}+\frac{4}{3} N_{r} \text {. }
$$

For the case $n_{1}=2 n^{\prime}+1$, there are 2 types of weird leptons. Of course more complicated flavor raids are possible, but enumerating them is probably academic.

\section{Case 5:}

The spinor representations of $\mathrm{SO}_{n}$ take the form, Eqs. (2.22) or (2.24) and (2.25)

$$
\mathbf{f}=\left(\mathbf{L}, \mathbf{1}^{c}\right)+\left(\mathbf{q}, 3^{c}\right)+\left(\overline{\mathbf{r}}, \overline{\mathbf{3}}^{c}\right)+\left(\overline{\mathbf{1}}, \mathbf{1}^{c}\right),
$$

where $\bar{\Gamma}$ and $\overline{\mathbf{r}}$ transform as the $\xi$ spinor of $\mathrm{SO}_{n-6}$, and $\mathbf{q}$ and $L$ as the $\xi^{\prime}$ spinor. (We recall that $\xi$ and $\xi^{\prime}$ are equivalent for $n$ odd and conjugates of each other when $n$ is twice an even number.) The explicit $\mathrm{U}_{1}$ ' $\mathrm{s}$ in Eqs. (2.20) and (2.23) are generated by

$$
X=N_{L}-\frac{1}{3} N_{q}+N_{l}-\frac{1}{3} N_{r},
$$

and combining Eq. (4.18) with $Z=N_{L}+N_{q}-N_{r}-N_{l}$, we find

$$
A=N_{L}+\frac{1}{3} N_{q}-\frac{2}{3} N_{r} .
$$

A flavor raid requires computing the eigenvalue spectrum of an arbitrary $\mathrm{U}_{1}$ in $\mathrm{SO}_{n-6}$, as we already have for the electric charge assignments in Case 5 of Sec. III. Following the notation established there the general solution for $N$ integer is

$$
\begin{aligned}
& A_{\alpha}\left(\xi, 1^{c}\right)=M_{+\alpha}+r+1, \\
& A_{\alpha}\left(\xi, 3^{c}\right)=M_{+\alpha}+s+1 / 3, \\
& A_{\alpha}\left(\xi^{\prime}, 1^{c}\right)=M_{-\alpha}+(3 s-r) / 2, \\
& A_{\alpha}\left(\xi^{\prime}, \overline{3}^{c}\right)=M_{-\alpha}+2 / 3+(r+s) / 2,
\end{aligned}
$$

where $r$ and $s$ must both be even integers or odd integers, and the fermion number

$$
Z=(r+3 s) / 4+1 / 2
$$

must be nonzero. The half-integer $N$ solution is obtained by replacing $r$ by $r-1 / 2$ and $s$ by $s-1 / 2$ everywhere in Eqs. (4.20) and (4.21).

\section{Case 6:}

The analysis of the vector representation of $S p_{2 n}$ is identical to Case 4 , except that $n_{1}$ cannot be odd.

\section{Case 7:}

$X$ must generate a $\mathrm{U}_{1}$ in $G_{q+l}$ for exceptional gauge groups. For any choice of this $U_{1}$, the proton stable $F_{4}$ theory that satisfies the restrictions we have made is flavor poor. The $U_{1}$ generator $X$ is a flavor $\mathrm{SU}_{3}$ generator, which is easily parametrized in a general way consistent with the constraints on $A$. The 26, which is doubled, has integer fermion number so that all the leptons in $\left(8,1^{c}\right)$ have integer $A$. Although this model has six quarks and eight leptons, it is straightforward to prove that if one (or more) lepton has $A=0$, then at least five of the six quarks are weird. Thus this $F_{4}$ model can only have one quark flavor.

\section{Case 8:}

The 27 of $E_{6}$ is complex. A general parametrization is

$$
\begin{aligned}
& A_{\alpha}\left(1,3,3^{c}\right)=n_{\alpha}-k+(M-2 N) / 3, \\
& A_{\alpha}\left(\overline{3}, 1, \overline{3}^{c}\right)=-m_{\alpha}-k-(N-2 M) / 3, \\
& A_{\alpha}\left(3, \overline{3}, 1^{c}\right)=m_{i}-n_{j}-k, \quad i, j=1,2,3,
\end{aligned}
$$

where $n_{\alpha}, m_{\alpha}$, and $k$ are integers, $N=n_{1}+n_{2}+n_{3}, M=m_{1}$ $+m_{2}+m_{3}$, and fermion number $Z$ is

$$
Z=(M-N) / 3-k \text {. }
$$

The sums $M$ and $N$ are restricted to:

(1) $M=0, N=1$ or $M=1, N=0$; and

(2) $M=N=2$ and $k \neq 0$.

The richest arrangement has six leptons with $A=0$ and four quarks with $A=1 / 3$ (three from one triplet). This is the solution with $n_{1}=n_{2}=n_{3}=0$, so that $X$ generates a $\mathrm{U}_{1}$ in just one of the $\mathrm{SU}_{3}$ factors. Other solutions with at least one nonweird lepton have at most three quarks with $A=1 / 3$.

\section{Case 9:}

The $E_{7}$ doubled 56 of fermions, Eq. (2.36), has 12 quarks; $X$ must generate a $\mathrm{U}_{1} \subset \mathrm{SU}_{6} \cdot$ As in Case 9 of Section III, we parametrize the $U_{1}$ in terms of six integers $\mathrm{n}$. Then there are two solutions

$$
\begin{aligned}
& A_{\alpha}\left(6,3^{c}\right)=n_{\alpha}+k+1 / 3, \\
& A_{\alpha}\left(\overline{6}, \overline{3}^{c}\right)=-n_{\alpha}+k+2 / 3, \\
& A_{\alpha}\left(20,1^{c}\right)=\Re_{3 \alpha}+k,
\end{aligned}
$$

where $k$ is any integer, $N=\sum n_{\alpha}=1$, and $\mathfrak{T}_{3}$ is the set of 20 threefold sums of $n_{\alpha}$. The other solution is

$$
\begin{aligned}
& A_{\alpha}\left(6,3^{c}\right)=n_{\alpha}+k-2 / 3, \\
& A_{\alpha}\left(\overline{6}, \overline{3}^{c}\right)=-n_{\alpha}+k+2 / 3, \\
& A_{\alpha}\left(20,1^{c}\right)=\Re_{3 \alpha}+k-2,
\end{aligned}
$$

where $k$, which is fermion number, is a nonzero integer and $N=4$. Thus we see that $X$ cannot generate the $U_{1}$ in $\mathrm{SU}_{6} \supset \mathrm{SU}_{3} \times \mathrm{SU}_{3} \times \mathrm{U}_{1}$, and that the $\mathrm{SU}_{6} \supset \mathrm{SU}_{4} \times \mathrm{SU}_{2} \times \mathrm{U}_{1}$ theory has only two nonweird quarks. The solution with the least number of weird fermions is Eq. (4.24) with $\left\{n_{\alpha}\right\}$ $=\{1,0,0,0,0,0\}$ and $k=0$. This corresponds to $X$ generating the $\mathrm{U}_{1}$ in $\mathrm{SU}_{6} \supset \mathrm{SU}_{5} \times \mathrm{U}_{1}$ 。 There are then five nonwèird $q$ quarks and one weird $s$ quark in the $\left(6,3^{\circ}\right)$, and five weird $\bar{r}$ antiquarks and one nonweird $\bar{\sigma}$ in the $\left(\overline{6}, \overline{3}^{c}\right)$. Half the leptons are weird. $A$ is given by

$$
A=N_{L}+N_{q} / 3+N_{\sigma} / 3+4 N_{s} / 3-2 N_{r} / 3 .
$$

This is the only example (without introducing more fermion representations) of a proton-preserving exceptional gauge theory with enough flavors for good phenomeno$\log$.

\section{B. Single $f$, doubling and breaking elsewhere}

In this set of solutions we assign all the fermions to a single irreducible self-conjugate representation of $G$, such as can be found in Cases $2,4,5,6,7$, and 9 of Table I. (We continue to follow the numbering system of Sec. II.) The global symmetry operator $Z$ has zero eigenvalue for the fermion representation, and $Z$ and $X$ are 
broken in some other sector of the theory such as the scalar-pseudoscalar sector, so $A=X$ for the fermions 。 The problem is reduced to studying local $U_{1}^{\prime}$ 's that are not generated by electric charge.

\section{Case 2:}

The self-conjugate $\mathrm{SU}_{n}$ representation $\left(2 \mathrm{k}^{k}\right)_{A}, \mathrm{Eq} \cdot(2.11)$ with $n=2 k$, need not be doubled, but a flavor raid is needed if there are to be any $A=0$ leptons. Equation (4.11) is the general solution for $A$, and for this case, Eq. (4.12) is modified to read

$$
r=M+3 s+1 \text {. }
$$

No further constraints arise from particle conjugation in f. A simple example is obtained with $\left\{n_{\alpha}\right\}$ $=\{1,0, \ldots, 0\}, M=1$. The existence of leptons with $A=0$ imposes $r=s=-1$. Here $X$ is the generator of the $U_{1}$ in the embedding $\mathrm{SU}_{n-3} \supset \mathrm{SU}_{n-4} \times \mathrm{U}_{1}$.

\section{Cases 4 and 6:}

The discussions of the self-conjugate vector representations of $\mathrm{SO}_{n}$ and $S p_{2 n}$ are identical. The generator of the explicit $U_{1}$, Eqs. (2.19) and (2.28), simply counts the number of $3^{c} \mathrm{~s}$ minus the number of $\overline{3}^{c}$ s. Therefore.

$$
A=N_{q} / 3 \text {. }
$$

When it is not possible to break this $U_{1}$ in another sector of the theory, then a flavor raid is necessary and implies weird particles.

\section{Case 5:}

The generator of the natural $U_{1}$ counts both $1^{c}$ and $3^{c}$ in the spinor representations of $\mathrm{SO}_{n}$, so it must be combined with an $\mathrm{SO}_{n-6}$ generator to obtain some $A=0$ leptons. (Only the $\mathrm{SO}_{2 n+1}$, all $n$, and $\mathrm{SO}_{2 n}, n$ even, spinors are self-conjugate.) Fermion number is zero, so $r=-2$ $-3 s$ from Eq. (4.21), which automatically satisfies the antiparticle conjugation constraints. A simple example of this general solution, given by $\left\{n_{\alpha}\right\}=\{1,0, \ldots, 0\}$, corresponds to $X$ generating the $\mathrm{U}_{1}$ in $\mathrm{SO}_{n-6} \supset \mathrm{SO}_{n-8} \times \mathrm{U}_{1}$. There are equal numbers of leptons with $A=0$ and $A=1$, and quarks with $A=1 / 3$ and $A=-2 / 3$.

\section{Case 7:}

There are only three quark flavors in the particleantiparticle self-conjugate 26 of $F_{4}$, and $X$ must generate a $\mathrm{U}_{1}$ in the flavor $\mathrm{SU}_{3}$. Consequently there can be at most two nonweird quarks.

\section{Case 9:}

There are six quarks and ten leptons in the 56 of $E_{7}$. There is only one solution with more than three nonweird leptons or more than three nonweird quarks: $X$ generates the $\mathrm{U}_{1}$ in $\mathrm{SU}_{6} \supset \mathrm{SU}_{4} \times \mathrm{SU}_{2} \times \mathrm{U}_{1}$. Then there are four quarks with $A=1 / 3$ and six leptons with $A=0$.

\section{ACKNOWLEDGMENTS}

We wish to thank many friends and colleagues at LASL, Aspen Center for Physics, and Caltech for stimulating conversations, especially G. Stephenson, T. Goldman, and $R$. Roskies. We gratefully acknowledge the hospitality of the Aspen Center for Physics, where some of the work was performed. The typing of several drafts of this paper was cheerfully and excellently done by Marian Martinez. We also wish to thank the CERN Theory Division Secretariat for kindly assisting us in preparing the final draft.

\section{APPENDIX}

We state and prove here the theorem that justifies the embedding procedure followed in Sec. II. Consider any embedding of $\mathrm{SU}_{3}^{c}$ in a simple Lie group $G$ for which there is at least one representation $f$ with color content restricted to $1^{c}, 3^{c}$, and $\overline{3}^{c}$. Then the fundamental representation of $G$ must also be limited to $1^{c}, 3^{c}$, and $\overline{3}^{c}$. In other words, the condition that the fermion representation contains color singlets, triplets, and possibly antitriplets implies Eq. (2.2) for the fundamental representation. The fundamental representations of the simple Lie groups are: $\mathrm{n}$ of $\mathrm{SU}_{n} ; \mathrm{n}$ of $\mathrm{SO}_{n} ; 2 \mathrm{n}$ of $S p_{2 n} ; 7$ of $G_{2} ; 26$ of $F_{4} ; 27$ of $E_{6} ; 56$ of $E_{7} ;$ and 248 of $E_{8}$.

The proof for the classical groups merely requires finding the color content of the group generators, which is explicitly displayed by the adjoint representation. Let $\mathrm{c}$ be a set of generators forming an irreducible representation of $\mathrm{SU}_{3}^{c}$. Since each group generator must transform $f$ within the representation, it is necessary that $\mathrm{c}$ acting on any $\mathrm{SU}_{3}^{c}$ representation in $\mathbf{f}$ contain at least one of the color representations in $f$. If $f$ has only color singlets, triplets, and antitriplets, then $\mathrm{c} \times 1^{c}$, $c \times 3^{c}$, or $c \times \overline{3}^{c}$ must contain a $1^{c}, 3^{c}$, or $\overline{3}^{c}$. This is true only if $\mathrm{c}$ is $1^{c}, 3^{c}, \overline{3}^{c}, 6^{c}, \overline{6}^{c}$, or $8^{c}$. Thus $\mathrm{f}$ can satisfy the color restriction only if each of the color representations in the adjoint $G$ has dimension less than or equal to 8 . The proof is completed by constructing the adjoint representation, which must satisfy this condition, from the fundamental representation.

Suppose the $\mathrm{n}$ of $\mathrm{SU}_{n}$ violates our color restriction, so that it contains a set of operators d transforming as some higher representation (dimension greater than 3 ) of $\mathrm{SU}_{3}^{c}$. The adjoint representation of $\mathrm{SU}_{n}$ is $\mathrm{n} \times \overline{\mathrm{n}}-1$, so it includes generators transforming under $\mathrm{SU}_{3}^{c}$ as the representations in $d \times \bar{d}$, which always contains a $27^{c}$. The theorem then follows for $\mathrm{SU}_{n}$. The proof is similar for the orthogonal and symplectic groups. If the $\mathrm{n}$ (vector representation) of $\mathrm{SU}_{n}$ contains a set $\mathrm{d}$ as defined above, then the adjoint representation, constructed from $(\mathrm{n} \times \mathrm{n})_{A}$, must include sets of generators that transform as the representations in $(\mathrm{d} \times \mathrm{d})_{A}$ under $\mathrm{SU}_{3}^{c}$. The theorem follows since $(\mathrm{d} \times \mathrm{d})_{A}$ always has at least one representation of dimension greater than 8 . The adjoint representation of $S p_{2 n}$ is constructed from $(2 n \times 2 n)_{s}$, and includes color operators in $(\mathrm{d} \times \mathrm{d})_{s}$. As before, $\mathrm{d}$ must be empty if $(2 n \times 2 n)_{s}$ is to have no sets of generators that transform under $\mathrm{SU}_{3}^{c}$ as a representation of dimension greater than 8 .

There exist embeddings of $\mathrm{SU}_{3}^{c}$ in the exceptional groups where the fundamental representation is not restricted to $1^{c}, 3^{c}$, and $\overline{3}^{c}$, but where the adjoint contains no color representations of dimension greater than 8 . The previous proof must then be supplemented with some information about the commutation relations of the group generators. We consider each exceptional group individually.

Three cases are trivial. Since the 7 of $G_{2}$ is self-con- 
jugate, it must decompose to $1^{c}+3^{c}+\overline{3}^{c}$. The decomposition of $E_{6}$ into $\mathrm{SU}_{3} \times \mathrm{SU}_{3} \times \mathrm{SU}_{3}$ is essentially symmetrical, and any $\mathrm{SU}_{3}$ may be color, as is clear from Eqs. (2.32) and (2.34). $E_{8}$ is hopeless since the fundamental representation is the adjoint, which must have an $8^{c}$.

We might use the other $\mathrm{SU}_{3}$ of Eq. (2.29) as the color subgroup of $F_{4}$. The adjoint representation is then

$$
52=\left(1,8^{c}\right)+\left(8,1^{c}\right)+\left(3, \overline{6}^{c}\right)+\left(\overline{3}, 6^{c}\right),
$$

as can be seen from Eq. (2.31). A color octet does appear in the fundamental representation, Eq. (2.30), for this embedding, so wé must prove that no higher representations satisfy our color restrictions. Consider the action of the generators transforming as $\left(3, \overline{6}^{c}\right)$ on the supposed higher representation of the form, $\left(y, 1^{c}\right)$ $+\left(x, 3^{c}\right)+\left(\bar{x}, \overline{3}^{c}\right)$, $x$ and $y$ being any representations of the flavor $\mathrm{SU}_{3}$. Since these generators annihilate the pieces transforming as $\left(y, 1^{c}\right)$ and $\left(\bar{x}, \overline{3}^{c}\right)$, they must not annihilate $\left(x, 3^{c}\right)$. However, we prove that they do. The generators in $\left(\overline{3}, 6^{c}\right)$ must annihilate $\left(x, 3^{c}\right)$. All the commutation relations among these generators yield generators in $\left(3, \overline{6}^{c}\right)$. Therefore the $\left(3, \overline{6}^{c}\right)$ generators must also annihilate $\left(x, 3^{c}\right)$ and the representation cannot be of the supposed form. This completes the theorem for $F_{4}$.

Suppose $\mathrm{SU}_{3}^{c}$ is embedded in the $\mathrm{SU}_{6}$ subgroup of $E_{7}$, Eq. (2.35). If the color is identified with one of the $\mathrm{SU}_{3}$ 's in $\mathrm{SU}_{6} \supset \mathrm{SU}_{3} \times \mathrm{SU}_{3} \times \mathrm{U}_{1}$, then the fundamental 56 of $E_{7}$, under the decomposition of $E_{7} \supset E_{6} \times U_{1}$, is $56=27+\frac{77}{27}$ $+1+1$. This has no higher color representations. The other possibility is to obtain the $\mathrm{SU}_{3}^{c}$ from $\mathrm{SU}_{6} \supset \mathrm{SU}_{2}$ $\times \mathrm{SU}_{3}^{c}$. Here, higher color representations do occur in the 56 , since the 20 of $\mathrm{SU}_{6}$ decomposes as $\left(2,8^{c}\right)+\left(4,1^{c}\right)$. Again, we must prove that no other $E_{7}$ representation satisfies the color restrictions. Under $\mathrm{SU}_{6} \supset \mathrm{SU}_{2} \times \mathrm{SU}_{3}^{c}$, the only $\mathrm{SU}_{6}$ representations restricted to $1^{c}, 3^{c}, \overline{3}^{c}$ are 1,6 , and $\overline{6}$; the $\mathrm{SU}_{3} \times \mathrm{SU}_{6}$ content of the supposed higher representation must be $(y, 1)+(x, 6)+(\bar{x}, \overline{6})$. The generators of $E_{7}$ include a set $(\overline{3}, 15)$, which annihilates $(\mathrm{y}, 1)$ and $(x, 6)$, since $15 \times 6$ does not have 1,6 , or $\overline{6}$. Moreover the set of generators formed by the cummutators in $(3, \overline{15})$ with itself also falls into the $(\overline{3}, 15)$ class, so that $(\overline{3}, 15)$ also annihilates $(\bar{x}, \overline{6})$. Thus there is no faithful representation of the supposed type.

\section{REFERENCES}

Abbott, L. F., M. Grisaru, and H. Schnitzer, 1977, to be published.

Adler, S. L., 1969, Phys. Rev. 177, 2426.

Bardeen, W. A., H. Fritzsch and M. Gell-Mann, 1972, in Scale and Conformal Symmetry in Hadron Physics, edited by $\mathrm{R}$. Gatto (Wiley, New York).

Bardeen, W. A., 1975, unpublished.

Bell, J. S., and R. Jackiw, 1969, Nuovo Cimento A 60, 47.

Brink, L., J. H. Schwarz and J. Scherk, 1977, Nucl. Phys. B 121, 77.

Curtright, T. L., 1977, Phys. Lett. B 71, 185.

de Rújula, A., H. Georgi, and S. Glashow, 1975, Phys. Rev. D 12,3589 .

Deser, S., and B. Zumino, 1976, Phys. Lett. B 62, 335.

Dynkin, E. B., 1957, Amer. Math. Soc. Trans. 3, 6, 111.

Englert, F., and R. Brout, 1964, Phys. Rev. Lett. 13, 321.

Fayet, P., 1976, unpublished.

Fayet, P., and J. Iliopoulos, 1974, Phys. Lett. B 51, 461.
Ferrara, S., 1976, Riv. Nuovo Cimento 6, 105.

Ferrara, S., and B. Zumino, 1974, Nucl. Phys. B 79, 413.

Freedman, D., P. van Nieuwenhuizen, and S. Ferrara, 197,6, Phys. Rev. D 13, 3214.

Fritzsch, H., and M. Gell-Mann, 1971, in Proceedings of the International Conference on Dualityand Symmetry in High Energy Physics, edited by E. Gotsman (Weizmann, Jerusalem). Fritzsch, H, and M. Gell-Mann, 1972, Proceedings of the XVI Intermational Conference on High Energy Physics, Vol. 2, p. 135. (National Accelerator Laboratory).

Fritzsch, H., M. Gell-Mann, and H. Leutwyler, 1973, Phys. Lett. B 74, 365 .

Fritzsch, H., M. Gell-Mann, and P. Minkowski, 1975, Phys. Lett. B 59, 256.

Fritzsch, H., and P. Minkowski, 1975, Ann. Phys. (N.Y.) 93, 193.

Gell-Mann, M., and S. L. Glashow, 1961, Ann. Phys. (N.Y.) 15, 437.

Georgi, H., 1975, in Particles and Fields-1974, AIP Conference Proceedings No. 23, Particles and Fields Subseries No. 10, Williamsburg, Va., edited by Carl E. Carlson (AIP, New York).

Georgi, H., and S. L. Glashow, 1973, Phys. Rev. D 6, 429.

Georgi, H., and S. L. Glashow, 1974, Phys. Rev. Lett. 32, 438. Georgi, H., H. Quinn, and S. Weinberg, 1974, Phys. Rev. Lett. $33,451$.

Gildener, E., 1976, Phys. Rev. D 14, 1667.

Gilmore, R., 1974, Lie Groups, Lie Algebras and Some of

Their Applications, (Wiley, New York).

Glashow, S., 1959, Ph.D. thesis, Harvard University.

Gliozzi, F., J. Scherk, and D. Olive, 1977, Nucl. Phys. B 122, 253.

Goldstone, J., 1961, Nuovo Cimento 19, 154.

Goldstone, J., A. Salam, and S. Weinberg, 1962, Phys. Rev. 127, 965.

Gol'fànd,. Y. A., and E. P. Likhtman, 1971, Zh. Eksp. Teor.

Fiz. Pis. Red. 13, 452. (English translation 13, 323.)

Greenberg, O. W., 1964, Phys. Rev. Lett. 13, 598.

Gross, D., and F. Wilczek, 1973, Phys. Rev. Lett. 30, 1343.

Günaydin, M., and F. Gürsey, 1973, J. Math. Phys. 14, 1651.

Guralnik, G. S., C. R. Hagen, and T. W. B. Kibble, 1964, Phys. Rev. Lett. 13, 585.

Gurr, H. S., W. R. Kropp, F. Reines, and B. Meyer, 1967, Phys. Rev. 158, 1321.

Gürsey, F., 1976, in New Pathways in High-Energy Physics $I I$, Proceedings of Orbis Scientiae 1976, Coral Gables, Florida, edited by Arnold Perlmutter (Plenum, New York).

Gürsey, F., P. Ramond, and P. Sikivie, 1975, Phys. Lett. B $60,177$.

Gürsey, F., and P. Sikivie, 1976, Phys. Rev. Lett. 36, 775.

Gürsey, F., and P. Sikivie, 1977, Phys. Rev. D 16, 816.

Han, M., and Y. Nambu, 1965, Phys. Rev. B 139, 1006.

Hawking, S., 1975, Commun. Math. Phys. 43, 199.

Higgs, P. W., 1964a, Phys. Rev. Lett. 12, 132.

Higgs, P. W., 1964b, Phys. Rev. Lett. 13, 508.

Jones, D. R. T., 1975, Nucl. Phys. B 87, 127.

Jones, D. R. T., 1977, CERN preprint.

Kibble, T. W. B., 1961, J. Math. Phys. 2, 212.

Kingsley, R., S. Treiman, F. Wilczek, and A. Zee, 1975, Phys. Rev. D 12, 2768.

Nambu, Y., 1966, in Preludes in Theoretical Physics, edited by A. de Shalit (North-Holland, Amsterdam).

Nambu, Y., and G. Jona-Lasinio, 1961a, Phys. Rev. 122, 345; and Nambu, Y., and G. Jona-Lasinio, 1961b, Phys. Rev. 124, 246.

Neveu, A., and J. H. Schwarz, 1971, Nucl. Phys. B 31, 86.

Pakvasa, S., W. A. Simmons, and S. F. Tuan, 1975, Phys. Rev. Lett. 35, 702 .

Patera, J., and D. Sankoff, 1973, Tables of Branching Rules for Representations of Simple Lie Algebras (L'Université de Montréal, Montréal).

Pati, J. C., and A. Salam, 1973, Phys. Rev. D 8, 1240. 
Pati, J. C., and A. Salam, 1974, Phys. Rev. D 10, 275. Poggio, E., and H. Pendleton, 1977, Brandeis preprint. Politzer, H. D., Phys. Rev. Lett. 30, 1346. (1973).

Ramond, P., 1971, Phys. Rev. D 3, 2415.

Ramond, P., 1976, Nucl. Phys. B 110, 214.

Ramond, P., 1977, Nucl. Phys. B 126, 509.

Reines, F., and M. F. Crouch, 1974, Phys. Rev. Lett. 32, 493.

Salam, A., 1968, in Elementary Particle Theory, edited by

N. Svartholm (Almquist and Wiksell, Stockholm).

Salam, A., and J. Strathdee, 1974, Nucl. Phys. B 80, 499.

Salam, A., and J. Strathdee, 1975, Phys. Rev. D 11, 1521.

Scherk, J., and J. H. Schwarz, 1974, Nucl. Phys. B 81, 118.

Scherk, J., and J. H. Schwarz, 1975, Phys. Lett. B 57, 463.
Shaw, R., 1955, The Problem of Particle Types and Other Contributions to the Theory of Elementary Particles, Cambridge $\mathrm{Ph} . \mathrm{D}$. thesis, unpublished.

't Hooft, G., 1971, Nuc1. Phys. B 35, 167.

't Hooft, G., 1976, Phys. Rev. Lett. 37, 8.

Utiyama, R., 1956, Phys. Rev. 101, 1597.

Weinberg, S., 1967, Phys. Rev. Lett. 19, 1264.

Weinberg, S., 1973a, Phys. Rev. Lett. 31, 494.

Weinberg, S., 1973b, Phys. Rev. D 8, 4482.

Wess, J., and B. Zumino, 1974, Phys. Lett. B 49, 54.

Wybourne, B. G., 1974, Classical Groups for Physicists (Wiley-Interscience, New York).

Yang, C. N., and R. L. Mills, 1954, Phys. Rev. 96, 191. 